\title{
ChandraStudy of a Complete Sample of Millisecond Pulsars in 47 Tucanae and NGC 6397
}

\section{Citation}

Grindlay, J. E., F. Camilo, C. O. Heinke, P. D. Edmonds, H. Cohn, and P. Lugger. 2002.

"ChandraStudy of a Complete Sample of Millisecond Pulsars in 47 Tucanae and NGC 6397." The Astrophysical Journal 581 (1): 470-84. https://doi.org/10.1086/344150.

\section{Permanent link}

http://nrs.harvard.edu/urn-3:HUL.InstRepos:41399879

\section{Terms of Use}

This article was downloaded from Harvard University's DASH repository, and is made available under the terms and conditions applicable to Other Posted Material, as set forth at http:// nrs.harvard.edu/urn-3:HUL.InstRepos:dash.current.terms-of-use\#LAA

\section{Share Your Story}

The Harvard community has made this article openly available.

Please share how this access benefits you. Submit a story.

Accessibility 
DRAFT VERSION NOVEMBER 2, 2018

Preprint typeset using LATEX style emulateapj v. 14/09/00

\title{
CHANDRA STUDY OF A COMPLETE SAMPLE OF MILLISECOND PULSARS IN 47 TUC AND NGC 6397
}

\author{
J. E. Grindlay ${ }^{1}$, F. CAMilo ${ }^{2}$, C. O. Heinke ${ }^{1}$, P. D. Edmonds ${ }^{1}$, H. Cohn ${ }^{3}$ And P. Lugger ${ }^{3}$ \\ Draft version November 2, 2018
}

\begin{abstract}
We report Chandra observations of the complete sample of millisecond pulsars (MSPs) with precise radio positions in the globular clusters 47 Tuc (NGC 104) and NGC 6397. The x-ray luminosities and colors are derived or constrained and compared to x-ray MSPs previously detected in the field as well as one previously detected in a globular cluster (M28). The 47 Tuc MSPs are predominantly soft sources suggestive of thermal emission from small $\left(\mathrm{r}_{x}<0.6 \mathrm{~km}\right)$ polar caps on the neutron star rather than magnetospheric emission and are a relatively homogeneous sample, with most $\mathrm{X}$-ray luminosities in a surprisingly narrow range $\left(\mathrm{L}_{x} \sim 1-4 \times 10^{30} \mathrm{erg} \mathrm{s}^{-1}\right)$. We use previously derived intrinsic $\dot{P}$ values and find a new relation between $\mathrm{L}_{x}$ and spindown luminosity, $\dot{E}: \mathrm{L}_{x} \propto$ $\dot{E} \beta$, with $\beta \sim 0.5 \pm 0.2$ vs. $\sim 1.0$ for both pulsars and MSPs in the field. Adding the single MSP in NGC 6397 constrains $\beta=0.5 \pm 0.15$. This $\mathrm{L}_{x}-\dot{E}$ relation and also the $\mathrm{L}_{x} / \dot{E}$ vs. spindown age are each similar to that found by Harding \& Muslimov (2002) for thermal emission from polar cap heating. However, the cluster MSPs are relatively longer-lived (in thermal x-rays) than either the models or field MSPs, which may have additional magnetospheric (non-thermal) components. We suggest the cluster MSPs may have altered surface magnetic field topology (e.g. multipole) or their neutron stars are more massive from repeated accretion episodes due to encounters and repeated exchange interactions. MSP binary companions on or just off the main sequence (e.g. NGC 6397) are likely to have been re-exchanged and might show anomalous $\dot{P}$ and $\dot{E}$ values due to relaxation of misaligned core-crust spins. The radial distribution of $\sim 40$ soft Chandra sources in 47 Tuc is consistent with a $\sim 1.4 M_{\odot}$ component in a multi-mass King model and with the identified MSP sample. The implied total MSP population in 47 Tuc with $\mathrm{L}_{x} \gtrsim 10^{30} \mathrm{erg} \mathrm{s}^{-1}$ is $\sim 35-90$, and can constrain the relative beaming in radio vs. soft x-rays. NGC 6397 is relatively deficient in MSPs; its single detected example may have been re-exchanged out of the cluster core.
\end{abstract}

Subject headings: globular clusters: general — globular clusters: individual (47 Tucanae) — stars: neutron — $\mathrm{X}$-ray: stars — binaries: general — pulsars: general

\section{INTRODUCTION}

Millisecond pulsars (MSPs) are end-products of binary evolution. In the Galactic disk, they are the fossil markers of rare primordial binaries in which neutron stars (NSs) are retained after birth in systems with low mass companions, from which accretion and a low mass x-ray binary (LMXB) phase transfers angular momentum to spin them up to msec periods. The "standard model" of MSPs as the remnants of LMXBs (reviewed by Bhattacharya \& van den Heuvel 1991) is supported by the discovery of the msec X-ray pulsar XTE J1808-369 (Wijnands \& van der Klis 1998) as well as the accumulating evidence for msec pulsation periods in a number of LMXBs from apparently stable frequencies in their quasi-periodic oscillation power spectra. However formation and evolution questions remain since no LMXB in quiescence (qLMXB) has yet been found as a radio MSP, and the reduction in angular momentum transfer efficiency imposed by likely advection dominated accretion flow (ADAF)-type accretion in quiescence means that the MSPs predominantly arise from a population of bright LMXB transients (Yi \& Grindlay 1998) which may not be observed in numbers sufficient to support the disk MSP population. The recent radio detection of 20 MSPs in the globular cluster 47 Tuc (NGC 104) by Camilo et al. (2000), and the subsequent $\mathrm{x}$-ray detection with Chandra (Grindlay et al. 2001a; hereafter GHE01a) of the 15 that were precisely located with radio timing solutions (Freire et al. 2001a; hereafter FCL01), have opened a new window for the study of the nature and formation/evolution of MSPs.

In globular clusters, in which MSPs are expected to show the same factor of $\sim 100-200$ excess in their numbers (per unit stellar mass) as do the LMXBs compared with the disk population (Clark 1975), the MSPs trace more exotic binary evolution (and possibly stellar evolution) paths than in the field. Here the MSP progenitor binaries can be formed readily from main sequence binaries by the exchange of a NS into the binary (e.g. Rasio, Pfahl, \& Rappaport 2000) or they may also (in very high density cluster cores) form from direct 2-body tidal capture (Mardling 1995) or 3-body encounters. Clusters also allow the exchange of the original mass donor with a replacement main sequence (or near turnoff, given mass segregation) star, for which the MSP discovered in NGC 6397 (D'Amico et al. 2001a; D'Amico et al. 2001b; hereafter DPM01; Ferraro et al. 2001) and detected with Chandra (Grindlay et al. 2001b; hereafter GHE01b) might be the first example.

The 15 MSPs with precise locations (FCL01) include 47 Tuc$\mathrm{G}$ and -I separated by just 0 !' 12 . The sample is now extended to 16 with the timing position for 47 Tuc-S (Freire 2001), which places it just $0 . / 7$ from 47 Tuc-F. We have identified (GHE01a) probable $\mathrm{x}$-ray emission from the original 15 MSPs (-G and -I

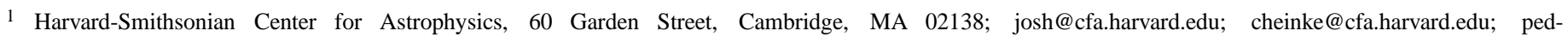
monds@cfa.harvard.edu

${ }^{2}$ Columbia Astrophysics Laboratory, Columbia University, 550 West 120th Street, New York, NY 10027; fernando@astro.columbia.edu

${ }^{3}$ Department of Astronomy, Indiana University, Bloomington, IN 47405; cohn@ astro.indiana.edu; lugger@astro.indiana.edu
} 
cannot be resolved, and $-\mathrm{C}$ is a marginal identification at best, as described below), and we report here the possible detection of 47 Tuc-S. In this paper, we provide the details for the Chandra detections of this complete (for radio detection) sample of MSPs and derive the x-ray properties of this first significant sample of MSPs detected in a single globular. We derive the X-ray luminosities, spindown energy $\operatorname{loss} \dot{E}$, and the characteristic age $(P / 2 \dot{P})$ for these MSPs, using the 47 Tuc distance, $4.5 \pm 0.3 \mathrm{kpc}$, derived from the recent compilation of Zoccali et al. (2001). This is the first derivation of these properties for a significant sample of MSPs at a common known distance. We also compare the 47 Tuc MSP results to those of the single MSP detected in NGC 6397 (GHE01b) and previous (ROSAT) results for MSPs in the field and one other globular (M28), as summarized in the review by Becker \& Trümper (1999; hereafter BT99).

\section{CHANDRA OBSERVATIONS AND ANALYSIS OF MSPS IN} 47 TUC

A $74 \mathrm{ksec}$ Chandra observation (72ksec total data obtained) was conducted on 2000 March 16-17, in which at least 108 sources were detected in the $2^{\prime} \times 2.5$ around the cluster center (GHE01a). With the ACIS-I detector used, the 0." 492 pixel size and $0 . \prime 7$ (FWHM) telescope-detector point spread function (psf) for sources within $\sim 4^{\prime}$ of on-axis combine to give source positions with $1 \sigma$ centroid uncertainties of $\lesssim 0$." 3 for sources detected with $\gtrsim 90 \%$ confidence (Jerius et al. 2000). Even a source identified with just 1 count (cf. discussion below of MSP 47 Tuc-C) is thus located with $\sim 0$.'5 $(1 \sigma)$ centroiding error. The uncertainties in the absolute positions of Chandra sources are dominated by aspect (guide star) offsets (i.e. boresight offsets between the X-ray and optical axes) and are currently estimated to be $\sim 0.7$ (Aldcroft et al. 2000).

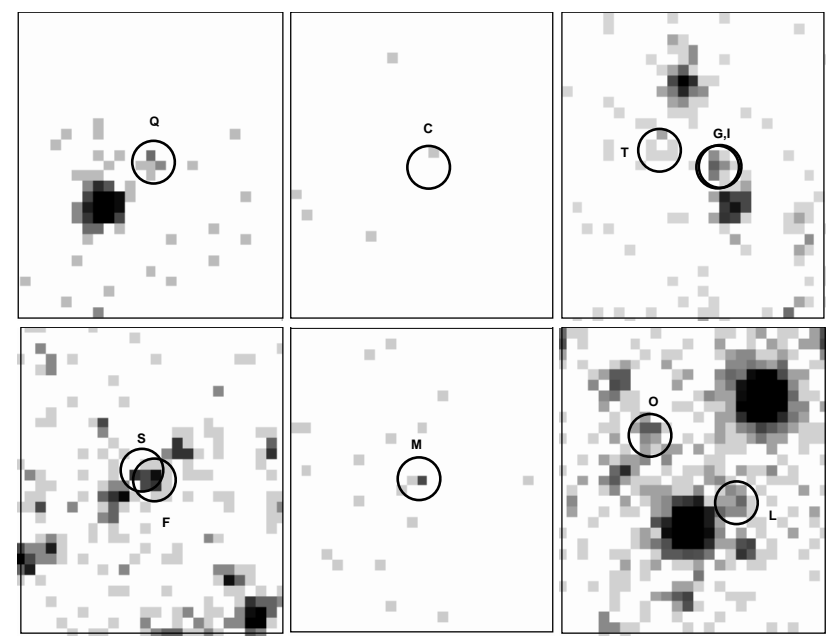

FIG. 1.- Images $(0.5-4.5 \mathrm{keV})$ of the MSPs in 47 Tuc not detected (or confused) by WAVDETECT (Q, C, T, G \& I, F \& S, and L), showing probable emission from the MSP in each case, and the weakest detection (M). Pixel randomization has been removed from the pipeline processing, and $1^{\prime \prime}$ circles are placed on the radio MSP locations. These circles are used to extract the counts for each MSP, as they contain $\sim 95 \%$ of the encircled energy for $\lesssim 0.3 \mathrm{keV}$ sources, which describe all of the MSP spectra except for -J (cf. Fig. 2).

Given these relative and absolute errors for source positions, the initial run of the CIAO ${ }^{4}$ tool WAVDETECT (Dobrzycki et al. 1999) in our general study (GHE01a) of the sources near the center of 47 Tuc detected 10 sources containing 11 of the

\footnotetext{
${ }^{4}$ All CXC software is available at http://asc.harvard.edu/ciao.
}

MSPs with $\sim 3-10$ mas positions as given by FCL01. A con-

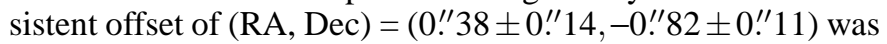
found for 6 relatively isolated MSPs (47 Tuc-D, -E, -H, -J, -N, -U) detected with WAVDETECT. Applying this offset to correct the Chandra positions to the MSP reference frame, we derived a small linear correction of the Chandra coordinates in both RA and Dec to minimize the rms deviations (with RA, Dec values 0 !'08, 0 !' 11 for the positions given in Table 1) with the radio positions. Details of this astrometry are given by Edmonds et al. (2001), who extended the astrometric solution to HST data to identify the likely optical counterpart for 47 Tuc-U. For the 9 remaining sources of FCL01, including the unresolved pair -G and -I, we used the precise astrometric solution to examine the Chandra images (in each band) for evidence of these MSPs (cf. Fig. 1). For 3 of these (47 Tuc-L, -Q, -T), WAVDETECT failed to detect the source due to crowding with a brighter neighbor, although inspection showed clumps of photons (similar in color and flux to the detected MSPs) centered at the radio position and consistent with the expected psf. For 47 Tuc-C only one count is detected at the predicted MSP position, but the relatively large exposure correction (factor of 2.5 [cf. Table 1], due to its being near a detector gap) for this source is consistent with it having X-ray flux within a factor of $\sim 2$ of the faintest other MSP, 47 Tuc-M.

Total counts were estimated for each MSP by extracting counts in each band in a weighted (by the psf) $1^{\prime \prime}$ radius cell centered on the MSP position and allowing for psf overlap contributions from neighboring sources using their WAVDETECT counts in each band and offset positions. The recently derived position for 47 Tuc-S (Freire 2001) shows that the original WAVDETECT flux (and thus derived x-ray colors and $\mathrm{L}_{x}$ ) for 47 Tuc-F (Table 1 of GHE01a) is contaminated by 47 Tuc-

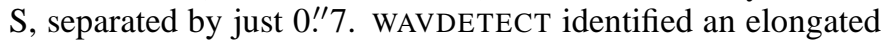
source between the positions of 47 Tuc-F and 47 Tuc-S, which can be best explained as the combined emission of the two sources. We estimate the counts for $-\mathrm{F}$ and $-\mathrm{S}$ by noting that the $\mathrm{X}$-ray position is twice as close to $-\mathrm{F}$ as $-\mathrm{S}$, and thus assign $-\mathrm{F}$ two-thirds of the counts. This is supported by visual inspection of the images in each band. Detected position offsets in the $0.5-$ $4.5 \mathrm{keV}$ primary detection band for the 10 WAVDETECT sources (12 MSPs) and counts in 3 bands (see below) for all 16 MSPs, are given in Table 1.

\subsection{X-ray Colors and Emission Models}

Our initial analysis of the MSP sample indicated the objects are relatively soft sources compared with the probable cataclysmic variables (CVs; GHE01a). In Table 1 we give the detected counts in 3 bands: softcts $(0.2-1 \mathrm{keV})$, mediumcts $(1-$ $2 \mathrm{keV}$ ) and hardcts (2-8 keV) for each of the 14 resolved MSPs, with counts for 47 Tuc-G and -I (unresolved) estimated. We use 3 bands rather than the hardness ratio Xcolor $=2.5 \log (\operatorname{cts}[0.5-$ $1.5 \mathrm{keV}] / \mathrm{cts}[1.5-6.0 \mathrm{keV}])$ used in GHE01a since it allows an approximate spectral analysis for the MSPs. With (limited) counts in 3 bands, we form the hardness ratios HR1 = mediumcts/softcts and HR2 = hardcts/mediumcts and plot the MSPs, with counting statistics errors, in the color-color diagram shown in Figure 2. The MSP colors are clustered in a relatively narrow range of HR1 and HR2; 47 Tuc-J is clearly (much) harder, as was evident in the Xcolor distributions plotted in GHE01a.

For comparison with the data, we construct (using the PIMMS tool for ACIS-I) values of HR1 and HR2 for 3 sim- 
ple models derived for the 47 Tuc absorption column $\left(N_{H}=\right.$ $2.4 \times 10^{20} \mathrm{~cm}^{-2}$; cf. GHE01a): thermal bremsstrahlung (TB), blackbody (BB) and power law (PL), with index values (kT or photon index) given in the caption of Figure 2. It can be seen that the observed range of HR1 vs. HR2 is roughly consistent with TB spectra with $\mathrm{kT} \sim 1 \mathrm{keV}$, BB spectra with $\mathrm{kT} \sim 0.2-$ $0.3 \mathrm{keV}$ or PL spectra with photon index $\sim 3$. The track for BB models is most consistent with the data for all but 47 Tuc-J. The weighted mean colors are plotted for all the MSPs and for all but 47 Tuc-J. This is consistent with a single $\mathrm{kT} \sim 0.22 \mathrm{keV}$ for all the MSPs (except -J). Thus we give in Table 1 approximate $\mathrm{x}$-ray luminosity values for a $\mathrm{BB}$ spectrum with $\mathrm{kT}=$ $0.22 \mathrm{keV}$. Apart from 47 Tuc-C, all other MSPs are detected with $\mathrm{L}_{x}$ in a surprisingly narrow range: $\sim 1-4 \times 10^{30} \mathrm{erg} \mathrm{s}^{-1}$, where the $\mathrm{L}_{x}$ values are again (as in GHE01a) quoted for the $0.5-2.5 \mathrm{keV}$ band to facilitate comparison with ROSAT results for MSPs (BT99) and to minimize uncertainties due to extrapolation of uncertain spectra over a broad band in which counts are not actually detected.

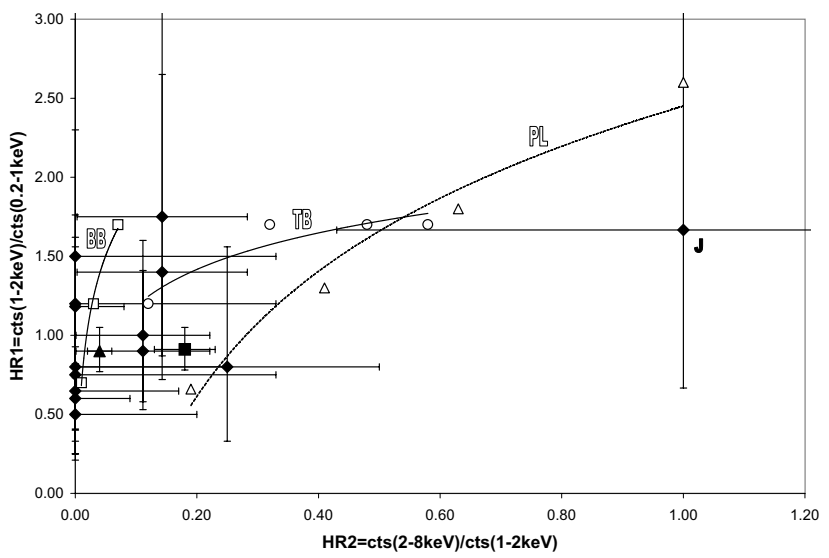

FIG. 2.-X-ray color-color diagram for all located MSPs (except 47 Tuc-C which is too faint, and -G which is combined with -I) in 47 Tuc (solid diamonds) vs. tracks for models with parameters changing from lower left to upper right: blackbody (BB; open squares), with $\mathrm{kT}=0.2,0.25,0.3 \mathrm{keV}$; power law (PL; open triangles), with photon index $\alpha=3,2,1.5,1$; and thermal bremsstrahlung (TB; open circles), with $\mathrm{kT}=1,2,3,6 \mathrm{keV}$. Weighted mean colors for the MSPs in 47 Tuc with and without 47 Tuc-J (labeled separately) are shown as the solid square and triangle, respectively.

Several constraints on MSP x-ray emission may be inferred from Figure 2. Given the average MSP luminosity (from Table 1) of $\mathrm{L}_{x} \sim 2 \times 10^{30} \mathrm{erg} \mathrm{s}^{-1}$, then for a BB with the suggested $\mathrm{kT} \sim 0.22 \mathrm{keV}$ or $\mathrm{T} \sim 2.6 \times 10^{6} \mathrm{~K}$, the bolometric luminosity is $\mathrm{L}_{x-b o l} \sim 3 \times 10^{30} \mathrm{erg} \mathrm{s}^{-1}$ and the total emitting radius is only $\mathrm{r}_{x} \sim 0.2 \mathrm{~km}$. NS H-atmosphere models, as needed for thermal spectra of the quiescent LMXBs in 47 Tuc (cf. Heinke et al. 2002 and references therein), would decrease the temperature by a factor of $\sim 2$ and thus increase the emission radius to $\sim 0.8$ $\mathrm{km}$. For a dipole field, both thermal (isotropic) polar caps are likely visible and the effective radius of each would be $\sim 0.14$ $\mathrm{km}$, or $\sim 0.56 \mathrm{~km}$ for $\mathrm{H}$-atmosphere models. Such a small emission area strongly suggests emission from a radiating area much smaller than the entire polar cap, which has expected radius of $\mathrm{r}_{p c}=\left(\Omega \mathrm{R}_{*} / \mathrm{c}\right)^{1 / 2} \mathrm{R}_{*}$ for a MSP with angular rotation frequency $\Omega$ and NS radius $\mathrm{R}_{*}$ (or $\mathrm{r}_{p c}=2.6 \mathrm{~km}$ for a $3 \mathrm{msec}$ period MSP and $\mathrm{R}_{*}=10 \mathrm{~km}$ ). Partially filled polar cap thermal emission (i.e. $\mathrm{r}_{x}<\mathrm{r}_{p c}$ ) was also found by Zavlin et al. (2002) for the bright MSP J0437-4715 (for which a two-component model could be fit to the much better statistics data than possible for the 47 Tuc MSPs) and is predicted by recent polar cap heating models of Harding \& Muslimov (2002; hereafter HM02), although we find important differences (cf. §5). The small emission area implies X-ray pulsations (which cannot be measured given the $3.2 \mathrm{sec}$ ACIS-I integration time) with a sinusoidal pulse shape appropriate to the fractional visibility of the isotropically radiating thermal polar cap(s). In contrast, the narrower pulse duty cycles of $\sim 10 \%$ for some field MSPs (and one in the globular cluster M28; cf. BT99) are probably due to non-thermal beamed emission.

If the $\mathrm{x}$-ray emission were a (pure) $\mathrm{TB}$ spectrum with $\mathrm{kT}$ $\sim 1 \mathrm{keV}$, the required emission measure EM $\sim 4 \times 10^{53} \mathrm{~cm}^{-3}$ would suggest plasma densities $\mathrm{n} \sim 3 \times 10^{26} \mathrm{R}^{-3 / 2} \mathrm{~cm}^{-3}$ for an emission region (assumed spherical) of radius $\mathrm{R}$. Such a region must not dominate the observed dispersion measure of the MSP, which is typically DM $=24.4 \mathrm{~cm}^{-3} \mathrm{pc}$ for the 47 Tuc MSPs with a variation $\delta \mathrm{DM} \sim 0.1 \mathrm{~cm}^{-3} \mathrm{pc}$ that is likely due to the ionized ISM in 47 Tuc (Freire et al. 2001b). Thus, the MSP local contribution to DM is probably $\mathrm{n} \cdot \mathrm{R} \lesssim 0.03 \mathrm{~cm}^{-3} \mathrm{pc} \sim 1$ $\times 10^{17} \mathrm{~cm}^{-2}$. Combined with the constraint above for EM, for a constant density emission region this implies an unrealistically large lower bound for the emission region: $\mathrm{R} \approx 3 \mathrm{pc}$. (For a TB source with radius $\mathrm{R} \sim 10^{10} \mathrm{~cm}$, or comparable to the size of the typical MSP binary separation, $\mathrm{n} \sim 3 \times 10^{11} \mathrm{~cm}^{-3}$ is required which would strongly violate the DM constraint.) Thus a pure TB spectrum is ruled out. Similarly, with the exception of 47 Tuc-J, PL models are unlikely since their photon index must be implausibly large $(\gtrsim 3)$ and are still inconsistent with the colors for most of the sample. The limited statistics can not, of course, rule out combined BB + PL models, but certainly it appears that the MSP sample in 47 Tuc is dominated by thermal emission from polar caps of the NSs.

\section{X-RAY VS. RADIO PROPERTIES OF MSPS IN 47 TUC}

The 47 Tuc MSPs are the first significant sample of MSPs at known distance with measured $\mathrm{x}$-ray and radio properties. Several long-standing pulsar questions can be addressed.

\subsection{X-ray vs. Pulsar Spin Properties}

Foremost is the correlation of x-ray luminosity and pulsar spindown luminosity $\dot{E}$, which is found for field MSPs (with much more uncertain distances) to scale as $\mathrm{L}_{x} \sim 10^{-3} \dot{E}$ (BT99). Here the globular cluster is both a hindrance and an interesting laboratory: the MSPs are accelerated in the cluster potential, thus biasing the observed values of $\dot{P}$ and hence $\dot{E}\left(\propto \dot{P} / P^{3}\right)$. FCL01 have modeled the variations of $\dot{P}$ among the 15 MSPs with precise locations (not including 47 Tuc-S) to derive constraints on both the cluster potential and the intrinsic $\dot{P}_{i}$ values for the MSPs. Tighter constraints on $\dot{P}_{i}$ can be obtained by assuming the cluster gas (detected from variations in DM) is uniform so that the observed DM value and projected radius give the 3D position in the cluster for each MSP (see Freire et al. 2001b). Assuming a King model for the cluster, and using the central velocity dispersion $\sigma_{v 0}=11.6 \mathrm{~km} \mathrm{~s}^{-1}$ (Meylan \& Mayor 1986), then gives the cluster acceleration term (cf. Figure 2 of Freire et al. 2001b) which is subtracted from the observed $\dot{P} / P$ to infer the intrinsic $\dot{P}_{i}$. Using a standard NS moment of inertia $I=10^{45} \mathrm{~g} \mathrm{~cm}^{2}$, we then derive $\dot{E}=4 \pi^{2} I \dot{P}_{i} / P^{3}$ for each MSP. Results are given in Table 1 and plotted vs. $\mathrm{L}_{x}$ in Figure 3. Uncertainties in the derived $\dot{E}$ values are typically $0.2-0.5$ in the log (though several are larger) but are not shown for clarity; uncertainties in $\log \left(\mathrm{L}_{x}\right)$ are typically 0.2 (see Table 
1). For comparison with all MSPs previously detected in $\mathrm{x}$ rays, we also plot values for 10 field MSPs and one each in the globular clusters M28 and NGC 6397. The data for the field MSPs and M28 (from which $\dot{E}$ values are derived) are summarized in the new compilation of data and distances in Table 2 whereas the NGC $6397 \mathrm{x}$-ray results are from GHE01b and are discussed in detail in $\$ 4$ below. We have repeated this analysis for variations in both cluster distance $(5.0 \mathrm{kpc} ;+1.5 \sigma)$ and central velocity dispersion $\left(13.0 \mathrm{~km} \mathrm{~s}^{-1} ;+1 \sigma\right)$ to sample the range of cluster acceleration models, and thus intrinsic $P_{i}$ values. Our goal is to derive the approximate index $\beta$ in the relation $\mathrm{L}_{x} \propto$ $\dot{E}^{\beta}$.

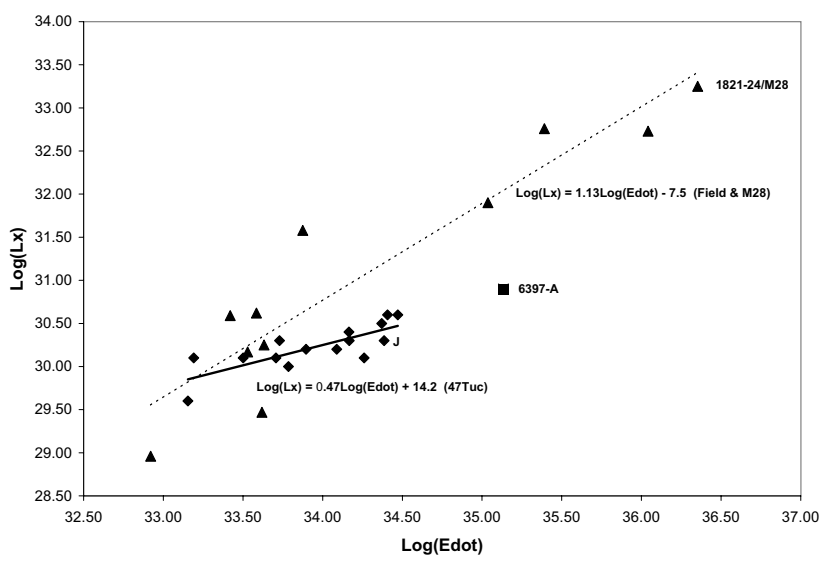

FIG. 3. $-\mathrm{L}_{x}$ vs. $\dot{E}$ for MSPs in 47 Tuc (diamonds) and field (triangles). MSP in NGC 6397 (square) is labeled as is -J in 47 Tuc. Values for MSPs in field and M28 are from Table 2, with $\mathrm{L}_{x}=4 \pi d^{2} F_{x}$ and $\dot{E}=4 \pi^{2} I \dot{P}_{i} / P^{3}$.

Whereas the field MSPs (and pulsars generally) are roughly consistent with the linear relation $\mathrm{L}_{x} \sim 10^{-3} \dot{E}$ (BT99), the MSPs in 47 Tuc appear to have a much weaker dependence: $\log \mathrm{L}_{x}=(0.47 \pm 0.10) \log \dot{E}+14.2 \pm 3.4$ for the nominal cluster model with $\sigma_{v 0}=11.6 \mathrm{~km} \mathrm{~s}^{-1}$, where the errors $( \pm 1 \sigma)$ are due to just the scatter in the points. Including the errors on each point, but with the approximation that unequal errors (on $\dot{E}$ ) are simply averaged (which biases the slope to steeper values, since the unequal errors are much larger for smaller values of $\dot{E}$ ), increases the slope to $0.59 \pm 0.28$ and offset to $10.0 \pm 10.6$. The best estimate of the slope is thus $\beta \sim 0.5 \pm 0.2$. Allowing for the error in the cluster model by using the $+1 \sigma$ value for the central velocity dispersion, $\sigma_{v 0}=13.0 \mathrm{~km} \mathrm{~s}^{-1}$, and again including the errors on each point gives slope $0.48 \pm 0.21$ and intercept $13.8 \pm 7.5$, and so is consistent. Similar variations in $\beta$ and the intercept are found for the increased (by about $1.5 \sigma$ ) cluster distance, $5 \mathrm{kpc}$. Thus, the MSPs in 47 Tuc have $\mathrm{L}_{x}(0.5-2.5 \mathrm{keV})$ values within a factor of $\sim 4$ (apart from 47 Tuc-C) despite a range of $\sim 25$ in $\dot{E}$. For comparison, the field (and M28) MSPs in Figure 3 have $\beta=1.13 \pm 0.15$ and offset $-7.48 \pm 5.03$ and so have $\beta$ consistent with both the ROSAT band $(0.1-2.4 \mathrm{keV})$ results of BT99 and the $2-10 \mathrm{keV}$ results derived by Possenti et al. (2002), who find $\beta=1.37 \pm 0.10$.

It is tempting to speculate these different $\beta$ values may be due to the different formation histories and possibly different physical parameters of MSPs in the field and 47 Tuc. In Figure $4 \mathrm{a}$ we plot $\mathrm{L}_{x}$ vs. spindown ages, $\tau=P / 2 \dot{P}_{i}$, for field and 47 Tuc MSPs. Error bars on the age parameter are not shown, for clarity, but are typically $\sim_{-0.1}^{+0.3}$ in the log and primarily due to the cluster acceleration model. Despite the uncertainties, the correlation is striking: the field MSPs show a declining $\mathrm{L}_{x}$ with "age," and the 47 Tuc MSPs appear to fall on this trend but with a flatter $\mathrm{L}_{x}$ vs. age slope. (Although both age and $\dot{E}$ are derived from combinations of $P$ and $\dot{P}_{i}$, they are algebraically different so that the same flatter correlations with $\mathrm{L}_{x}$ are not inevitable.) In $\S 5$ we compare the $\mathrm{L}_{x} / \dot{E}-\tau$ relation (Fig. $4 \mathrm{~b}$ ) with the models of HM02, finding both qualitative similarities (noted by HM02) but also important differences.
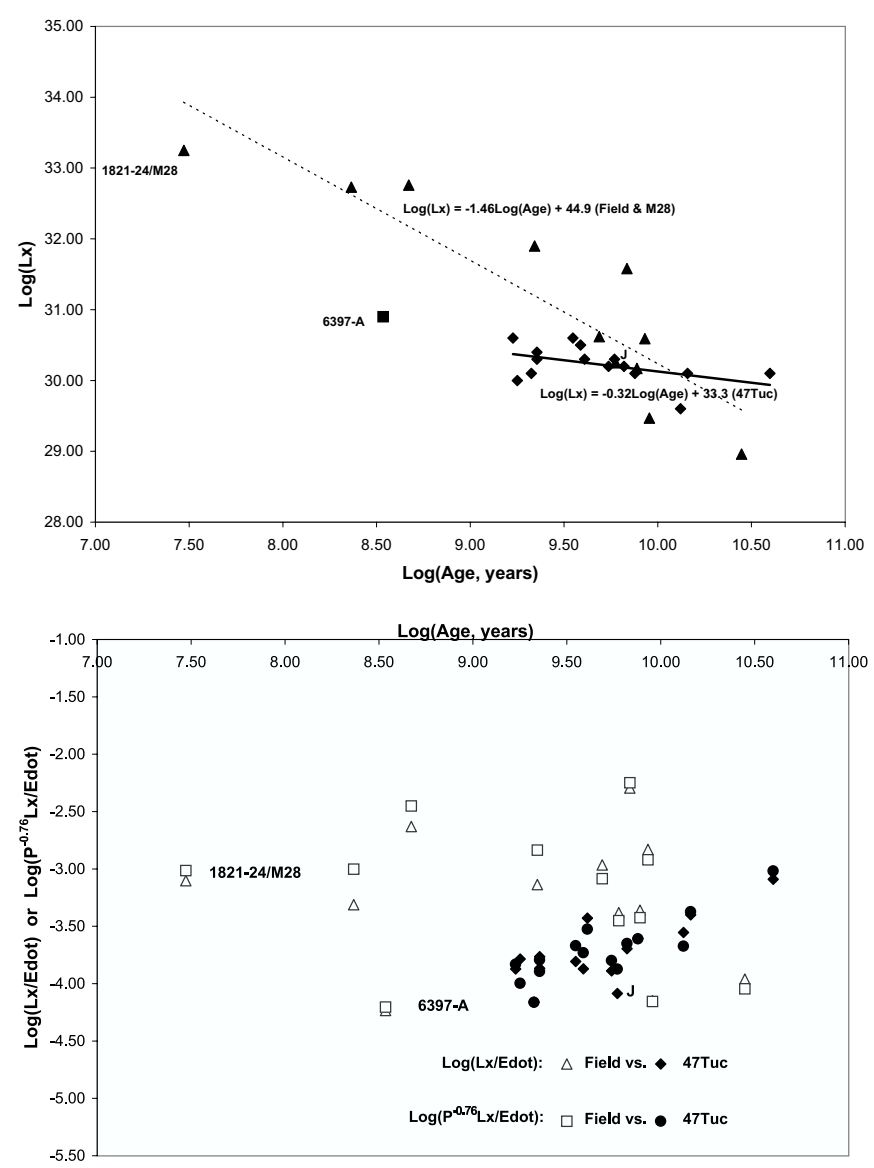

FIG. 4.- Top (a): $\mathrm{L}_{x}$ vs. spindown age $\left(\tau=P / 2 \dot{P}_{i}\right)$ for MSPs in 47 Tuc (diamonds) and field (triangles). MSP in NGC 6397 (square) is labeled as is -J in 47 Tuc. Values for MSPs in field and M28 are from Table 2. Bottom (b): $\mathrm{L}_{x} / E$ vs. spindown age, and approximate correction for pulse period $P$ (from HM02; see text for empirical dependences on $P$ and $\tau$ ) for 47 Tuc vs. field (and NGC 6397) MSPs.

We note that although spindown ages correspond only approximately to actual ages, the derived values are consistent with the formation of most 47 Tuc MSPs in a "burst" early in the cluster history. However this by itself would not provide an explanation for the different correlations observed for $\mathrm{L}_{x}$ in 47 Tuc and field MSPs. As a measure of the systematic uncertainties in age, the $0.6 \mathrm{Gyr}$ value derived from the cooling age for the He-WD companion of 47 Tuc-U (Edmonds et al. 2001) may be compared to the $\sim 0.9 \mathrm{Gyr}$ or $2 \mathrm{Gyr}$ spindown age of the MSP (two solutions for $\dot{P}_{i}$ are possible for 47 Tuc-U, as is also the case for $-\mathrm{G}$ and $-\mathrm{M}$ from the total sample of 15 for which the cluster acceleration model yields values for $\dot{P}_{i}$ and thus $\dot{E}$ and $\tau)$.

Another possible physical difference between the 47 Tuc and field MSPs might be magnetic field strength at the NS surface and thus also the light cylinder (at which the corotation speed equals $c$ ), since this likely affects the relative importance of non-thermal (magnetospheric) emission. For an assumed 
dipole field, this is given by $B_{\mathrm{lc}}=9.35 \times 10^{5} P_{i}^{1 / 2} P_{\mathrm{msec}}^{-5 / 2} \mathrm{G}$, with $\dot{P}_{i}$ in units of $10^{-20} \mathrm{~s} \mathrm{~s}^{-1}$. In Figure 5a we plot for the same pulsars $\mathrm{L}_{x}$ vs. $B_{\mathrm{lc}}$. As before, considered as a homogeneous group, the field pulsars (and M28) lie on a significantly steeper logarithmic slope (1.70 \pm 0.25$)$ than the 47 Tuc MSPs $(0.66 \pm 0.13)$. In contrast the field at the NS surface, $B_{\text {surf }}=3.2 \times 10^{19}(P \dot{P})^{1 / 2} \mathrm{G}$, is nearly independent of $\mathrm{L}_{x}$. The correlation of $\mathrm{L}_{x}$ with $B_{\text {surf }}$ (Fig. 5b) is less defined, with twice the scatter for both field and 47 Tuc MSPs, and with logarithmic slopes differing even more: $0.05 \pm 0.27$ for the 47 Tuc MSPs vs. $2.80 \pm 0.99$ for the field MSPs. Whereas at the light cylinder the dipole component of the field, $B_{\mathrm{lc}}$, would dominate, the surface field would be sensitive to multipole components which could give the larger scatter in Figure $5 \mathrm{~b}$. We return in $\S 5$ to additional arguments for a modified surface field topology.
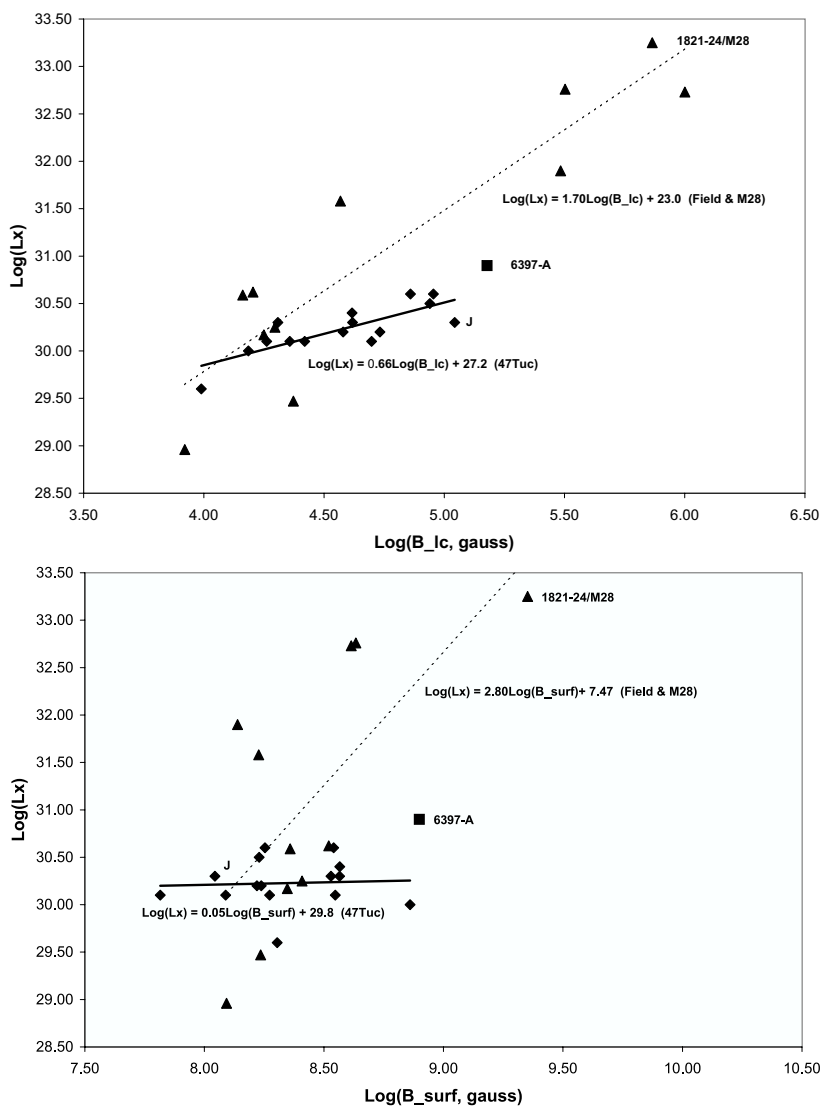

FIG. 5.- Top (a): $\mathrm{L}_{x}$ vs. $B_{\mathrm{lc}}$, inferred magnetic field strength at the light cylinder $\left(r_{\mathrm{lc}}=c P / 2 \pi\right)$ for MSPs in 47 Tuc (diamonds) and field (triangles). MSP in NGC 6397 (square) is labeled as is $-\mathrm{J}$ in 47 Tuc. Values for MSPs in field and M28 are from Table 2, with $B_{\text {lc }}$ derived assuming standard $1 / r^{3}$ dependence for a dipole field (see text). Bottom (b): $\mathrm{L}_{x}$ vs. surface magnetic field (see text).

We note that 3 out of the 4 MSPs in the field with $B_{\text {lc }} \gtrsim$ $10^{5.5} \mathrm{G}$ display $\mathrm{x}$-ray emission that is seemingly magnetospheric, with the nature of emission from the 4th (the eclipsing MSP B1957+20) indeterminate (BT99; Takahashi et al. 2001). Conversely, field pulsars with $B_{\mathrm{lc}}<10^{5} \mathrm{G}$ have x-ray emission that is typically either thermal or of indeterminate character (BT99). Considering the small numbers of such pulsars studied, and that most of them have highly uncertain distances (see Table 2), it is possible that field pulsars with $B_{\mathrm{lc}} \lesssim 10^{5} \mathrm{G}$ may show an $\mathrm{L}_{x}-B_{\mathrm{lc}}$ trend that is fairly flat and roughly consistent with the better determined relation for the 47 Tuc MSPs. However for this interpretation to hold, a few field MSPs with well determined distances (e.g. J0437-4715 and J1744-1134; cf. Table 2) must be accounted for and the even larger deviations of J0751+1807 and J1024-0719 from the 47 Tuc correlation line would require a factor $\gtrsim 3$ adjustments in these MSP distances. The fact that 5 of the 7 field MSPs with $\log (\dot{E}) \lesssim 34$ have $\mathrm{L}_{x}$ values above the trend-line for the 47 Tuc MSPs may suggest these field MSPs still have non-thermal emission components. We return (in $\S \S 5$ and 6 ) to further discussion of the possibility that the magnetic field configuration and evolution for old cluster NSs may differ from those in the field.

\subsection{Radial Distributions}

FCL01 pointed out the puzzling fact that the radial distribution of the (then) 15 MSPs with timing positions seemed to be truncated within $\sim 3.5 r_{c}$, where $r_{c}=24^{\prime \prime}$ is the core radius of the visible stars in the cluster as determined most recently (and completely) by Howell, Guhathakurta, \& Gilliland (2000). The addition of 47 Tuc-S, as the 16th MSP with a precise location (Freire 2001), does not change this. It is therefore of particular interest to compare the radial distribution of the 16 MSPs with the Chandra source distribution over a region large enough to measure the radial profile of the sources most likely to be MSPs. Our 2' $\times 2$ '.5 central field study (GHE01a) suggested most of the MSPs are soft and a possible total MSP population of $\sim 50$ in this region alone. Here we extend the study of the Chandra sources out to a radius of $4^{\prime}\left(\sim 10 r_{c}\right)$, to search for possible cutoffs or truncations in any of the Chandra source distributions as might be expected if the MSP distribution were cut off. We compare the MSP radial profiles with those of Chandra sources in several apparent spectral (hardness) classes to further test the possible identification of faint soft sources ("red background sources") with the large MSP population suspected (Camilo et al. 2000; GHE01a). This analysis will include, then, the vast majority of sources associated with 47 Tuc. Complete details (spectra, timing) of this source population will be included in the still-larger study (full ACIS $16^{\prime}$ field) of 47 Tuc to be reported by Heinke et al. (in preparation).

WAVDETECT analysis of the the $4^{\prime}$ (radius) field yields a total of 187 sources above a limit of medcts $(0.5-4.5 \mathrm{keV})=3$ (vs. 103 in GHE01a within the central $2^{\prime} \times 2$ '.5 box), or 126 sources above a 5 ct limit (vs. 92 in the $2^{\prime} \times 2^{\prime} .5$ box), which we impose to get $\mathrm{a} \geq 3 \sigma$ significance. For softness ratio (Xcolor $=2.5 \log [\operatorname{Soft}(0.5-1.5 \mathrm{keV}) / \mathrm{Hard}(1.5-6.0 \mathrm{keV})]$; cf. GHE01a) determination, we impose the WAVDETECT count limit of $3 \mathrm{cts}$ as an upper limit in either Soft or Hard bands if there is no WAVDETECT detection in that band. This alters the Xcolor values somewhat (for weak sources) from those given in GHE01a. In Figure 6a we provide the radial profiles of the sources detected with $\gtrsim 5$ cts in at least one band, which are divided into Soft (Xcolor $\gtrsim 1.0 ; 44$ sources) and Medium $(-1.0 \lesssim$ Xcolor $\lesssim 1.0 ; 41$ sources) groups. For the Medium group we also required that the source be detected by WAVDETECT ( $\gtrsim 3 \mathrm{cts})$ in both the soft $(0.5-1.5 \mathrm{keV})$ and hard $(1.5-6.0 \mathrm{keV})$ bands. Not plotted are the Hard (Xcolor $\lesssim-1.0 ; 9$ sources) sources and remaining sources (32) not included in the above 3 groups, since the Hard sources likely have a significant background AGN contribution (though at least 2 of these 9 sources are cluster CVs; GHE01a), and the remaining sources are indeterminate. The Medium source group, and thus its radial distribution, is also likely to be contaminated in this larger field by background AGNs. Scaling from the deep survey counts and AGN colors measured by Giacconi et al. (2000), we expect $\sim 8$ Medium 

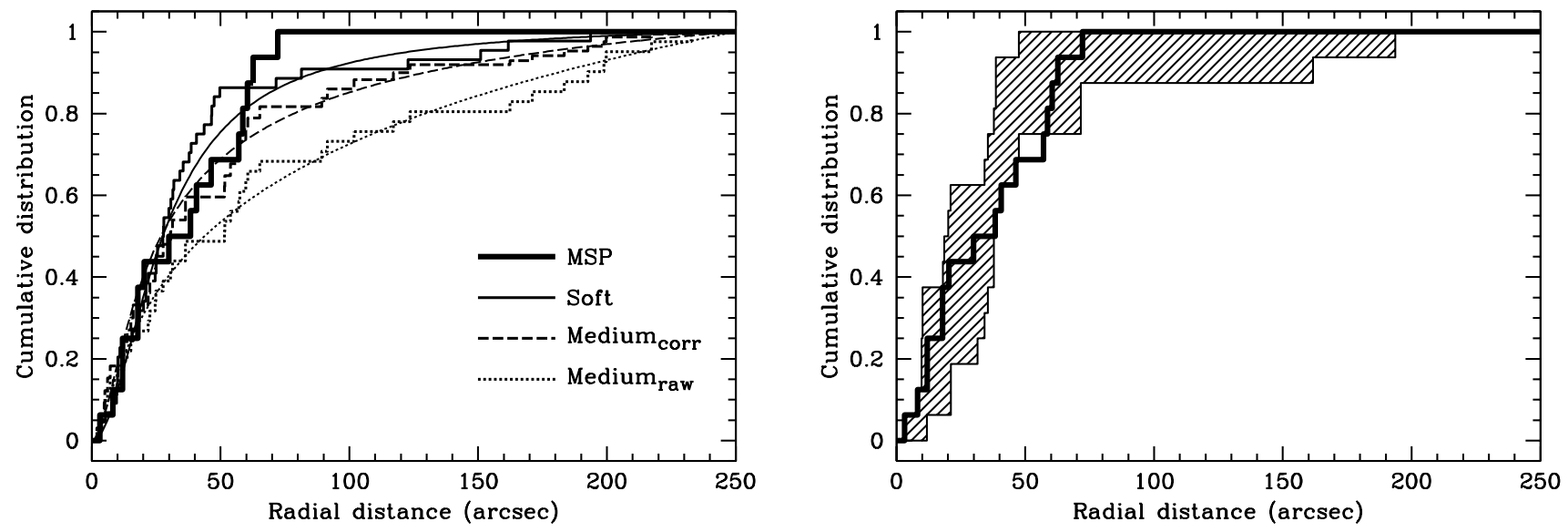

FIG. 6.- Top (a): radial source distributions for 47 Tuc MSPs vs. Chandra Soft (not including MSPs) and Medium (raw and with statistical subtraction of expected AGNs) sources detected with $\gtrsim 5$ cts together with best-fit King model predictions for each group. Bottom (b): envelope (5\% and $95 \%$ ) of radial distributions for 1000 samplings of 16 soft Chandra sources (hatched region) vs. the MSP sample (solid histogram).

and $\sim 3$ Hard sources to be background AGN. Therefore, the Medium source distribution has also (Fig. 6a) been corrected by the following Monte-Carlo procedure. A total of 1000 bootstrap resamplings of the distribution were generated. For each of these samples, an expected number of background sources was selected from a Poisson distribution with mean 8 . This number of radial source positions was then selected from a uniform spatial distribution (i.e. with cumulative distribution $\propto r^{2}$ ), and the closest actual sources to these positions were removed from the sample. The average of the 1000 background-corrected sample distributions was then adopted as the best estimate for the corrected cumulative distribution.

In Table 3 we give the probabilities from a KS test that each source group has a radial distribution consistent with that of any other group (including the 16-member MSP sample). For the Medium group, we show results for both the raw and corrected distributions. All of the cross-sample comparisons indicate consistency (i.e. probability of the samples being consistent exceeds 5\%) with the exception of the Medium-raw - Soft case. We ascribe this inconsistency to the contamination of the Medium-raw sample by background sources and note that the Medium-corrected sample is consistent with the Soft sample.

In Figure $6 \mathrm{~b}$ we show that 1000 repeated samplings of 16 sources from the Soft population gives a range of radial distributions that includes the observed MSP distribution. We note that both the CVs and BY Draconis (BY Dra) main sequence binaries have radial distributions that are also consistent with that of the Soft sources. However the radial incompleteness of our present HST sample and thus CV and BY Dra identifications makes this result preliminary.

For each of the observed or corrected (Medium) radial distributions in Figure 6a, we have fitted what we shall refer to as a "generalized King model" profile (cf. Lugger, Cohn, \& Grindlay 1995) to the projected surface density,

$$
S(r)=S_{0}\left[1+\left(\frac{r}{r_{0}}\right)^{2}\right]^{\alpha / 2} .
$$

Here $\alpha$ is the large- $r$ power-law index and the core radius $r_{c}$ is related to the radial-scale parameter $r_{0}$ by

$$
r_{c}=\left(2^{-2 / \alpha}-1\right)^{1 / 2} r_{0}
$$

The corresponding space density profile is given by

$$
n(r)=\frac{\Gamma\left(\frac{-\alpha}{2}\right)}{\sqrt{\pi} \Gamma\left(\frac{-\alpha-1}{2}\right)} \frac{S_{0}}{r_{0}}\left[1+\left(\frac{r}{r_{0}}\right)^{2}\right]^{\frac{\alpha-1}{2}} .
$$

Following the analyses of Jernigan \& Clark (1979), and Lightman, Hertz, \& Grindlay (1980), we assume that the projected distribution of the dominant visible stellar population, with mass $M_{*}\left(\sim 0.7 M_{\odot}\right.$ in the cluster core due to mass segregation), is described by an "analytic King model," i.e. the form given by eq. (1) with $\alpha_{*}=-2$. In thermal equilibrium, the space density of an x-ray source population with stellar mass $M_{x}=q M_{*}$ is given by $n_{x}(r) \propto n_{*}(r)^{q}$. Thus, the power-law index of the projected $\mathrm{x}$-ray source density profile is predicted to be

$$
\alpha_{x}=-(3 q-1)
$$

and the corresponding core radius is predicted to be

$$
\begin{aligned}
r_{c x} & =\left(2^{2 /(3 q-1)}-1\right)^{1 / 2} r_{c *} \\
& \approx 0.68\left(q-\frac{1}{3}\right)^{-1 / 2} r_{c *} .
\end{aligned}
$$

The latter approximation holds in the limit $q \gg 1$ and is accurate to $7 \%$ for $q \geq 2$.

In Table 4 we list the values derived for $r_{c}$ and $\alpha$ for each source group. The Soft sample is of particular interest, since it is the largest one $(N=44)$ and its parameters are reasonably well determined. As we have shown in Table 3, both the Medium-corrected and the MSP samples are consistent with the Soft sample. In principle, the mean x-ray source mass $M_{x}$ may be computed from eqs. (4) and (5). Eq. (4) gives $q=1.5$ for the best-fit value of $\alpha_{x}=-3.6 \pm 0.8$, with a $1-\sigma$ uncertainty range of $q=1.3-1.8$. Eq. (5a) gives $q=1.7$ for the best-fit value of $r_{c x}=15$ !' $^{\prime} \pm 3 . \prime 5$ together with $r_{c *}=24 . " 0$ (Howell et al. 2000), with a $1-\sigma$ uncertainty range of $q=1.3-2.5$. Thus, both measures of $q$ put the best-fit value at around 1.6 and allow values as large as $q \approx 2$. Given our estimate that the dominant mass in the core of 47 Tuc is $M_{*} \sim 0.7 M_{\odot}$, this implies that $M_{x}$ lies in the range $\sim 1.1-1.4 M_{\odot}$. For comparison, Grindlay et al. (1984) obtained a most probable value of $q=2.6$ for an ensemble of eight luminous LMXBs in as many clusters. 
There are a number of limitations to the "classic" analysis outlined above, which may well affect the estimate of the $\mathrm{x}$ ray source mass. First, this analysis assumes that the potential is dominated by stars of a single mass. In fact, there is a large range of stellar mass with substantial mass segregation. Secondly, the analysis assumes that the x-ray sources make a negligible contribution to the gravitational potential. However, given mass segregation, even a modest NS or heavy white dwarf (WD) population can make a substantial contribution to the potential in the innermost part of the cluster. Finally, the analysis assumes that the cluster is in a state of thermal equilibrium, i.e. that the cluster is isothermal and that each species is in energy equipartition. This condition does not hold rigorously, even in a nearly isothermal multi-mass King model. Also, the relatively low $M_{x} \sim 1.1-1.4 M_{\odot}$ may suggest non-equilibrium (perhaps from binary scattering) if the NS masses are $\gtrsim 1.4 M_{\odot}$ as possibly suggested by the long-lived thermal emission (cf. §5).

In light of these considerations, it is useful to compare our results for the Soft source distribution with the predictions from actual dynamical models. Meylan (1988) fitted multi-mass, anisotropic King-Mitchie models to surface brightness and stellar velocities for $47 \mathrm{Tuc}$, in order to constrain cluster parameters including the mass function. The models include a NS component, which comprises between $0.1-0.5 \%$ of the total cluster mass of $7 \times 10^{5} M_{\odot}$. Radial profiles for all components are given for one model with a NS mass fraction of $0.2 \%$ (equivalent to $\sim 1000 \mathrm{NSs}$ in the cluster). The power-law for the NS component has a slope of -4.0 in projection, which is consistent with the value of $\alpha=-3.6 \pm 0.8$ found here for the Soft source distribution. The predicted core radius for the NS component is $16^{\prime \prime}$, which is also in good agreement with our determination of $r_{c}=15$.' $^{\prime \prime} \pm 3$." $^{\prime \prime}$ for the Soft sources. By comparing the observed Soft source distribution with a set of models with a range of NS fraction, it should be possible to constrain this parameter. The models may be generated either by following Meylan's (1988) King-Mitchie model approach, or by Fokker-Planck integrations (e.g. Murphy et al. 1998). We are undertaking a study based on the latter method.

\subsection{Candidate MSP Sample in 47 Tuc and Estimated Total}

The KS probabilities (Table 3) and King model fits (Table 4) show that the radial distribution of the 44 Soft sources is indistinguishable from that of the MSPs, and indeed 7 of the 9 sources identified with MSPs with well-measured Xcolor values are soft. These soft MSPs include the doubles (-G, -I) and (-F, $-\mathrm{S})$, for which separate Xcolors could not be determined, so that in fact 9 MSPs are accounted for. The remaining MSPs are either faint (-M, -T; softcts $\lesssim 5$; cf. Table 1 ) or crowded (-L, -Q), but it can nevertheless be seen from the x-ray colors plotted in Figure 2 that all 47 Tuc MSPs, with the exception of 47 Tuc-J, are probably Soft (although 47 Tuc-U, with Xcolor = 0.9 , is formally in the medium group, while colors for 47 Tuc-C could not be measured).

Motivated by these results we have searched the list of Soft sources for extra MSP candidates. Of the 37 Soft sources not identified as MSPs, two are likely qLMXBs (X5 and X7; GHE01a and Heinke et al. 2002), two are CVs (V3 and AKO 9; GHE01a) and three are sources with counts more than two times brighter than the brightest detected MSP. Only 17 of the remaining 30 sources fall in the HST field of view (FOV) of Albrow et al. (2001). Nine of these 17 sources are astrometrically matched to optical variables (hereafter "active binary" candidates) identified with HST (Edmonds et al., in preparation), and an additional 2 are faint blue stars and possible CVs, one of which is also a periodic variable. Seven of the optical variables have independently been identified as likely BY Dras, red stragglers, or contact/semi-detached binaries by Albrow et al. (2001), and 2 of them were reported by GHE01a as BY Dras based on the additional (more restrictive) constraint of significant $\mathrm{X}$-ray variability.

Despite the lack of detection of any of the 7 soft MSPs as optical variables, it is possible that several of the active binary candidates contain MSPs. For example the optical companion to the one MSP known in NGC 6397 was previously identified as a BY Dra candidate by Taylor et al. (2001; hereafter TGE01). The corresponding Chandra source is relatively hard (see $\S 4$ ) and would not have been included in our list of soft sources in 47 Tuc, but we cannot be sure that all other MSPs with main sequence (or red straggler, as likely for the NGC 6397 MSP) companions will be similarly hard. In fact, after the initial submission of this paper, the relatively hard Chandra source W29 (GHE01b) has been identified with the MSP 47 Tuc-W as having a main sequence companion (Edmonds et al. 2002; cf. § 4). Also, one or both of the soft sources with a faint blue optical counterpart could be a He WD companion to an MSP (like 47 Tuc-U; Edmonds et al. 2001), instead of a CV. The definitive MSP characteristic is, of course, a pulsed radio source. However, a significantly larger sample of MSPs with radio timing positions, for comparison with the optical variables, will be difficult to obtain because so much observing time has been devoted to finding the 16 already known. Therefore, to estimate the number of MSPs implied by our soft sample we consider two extreme cases: (a) none of the candidate optical counterparts ( 9 active binary and $2 \mathrm{CV}$ candidates) are MSPs, or (b) all of the candidate optical counterparts are MSPs. For case (a) we find that only 6 of the soft sources falling in the HST FOV (and with counts $<2 \times$ the brightest detected MSP) do not have a known ID. Correcting for the detected fraction of MSPs with timing positions that are soft sources (7/16) and the incomplete FOV of HST (17/44 sources are outside the HST field) we estimate an extra population of $19(=6[1+17 / 44] \times 16 / 7)$ MSPs with $\mathrm{L}_{x} \gtrsim 10^{30} \mathrm{erg} \mathrm{s}^{-1}$, in addition to the 16 already known. For case (b) a total population of $69(=30 \times 16 / 7)$ extra MSPs is implied, for a total maximum MSP population of 85 .

These estimates are based on scaling from the number of soft source detections with WAVDETECT to the total MSP population with timing positions. Using a different technique we estimate the number of soft sources missed by WAVDETECT by examining the net counts not attributable to WAVDETECT sources within a radius of $24^{\prime \prime}$ (=1 $r_{c}$ for the background stars), where crowding will most limit faint source and crowded-field detection, and the annulus $1-2 r_{c}$ surrounding the cluster center. By removing counts from the original image in $1^{\prime \prime}$ radius regions around each WAVDETECT source (from GHE01a) in both the Softcts and Mediumcts bands (cf. Table 1), and then subtracting a fraction of the remaining counts (in each band) to correct for the psf-spillover from these bright(er) sources due to the energy-dependent Chandra telescope psf, we find $180 \pm 56$ Softcts, $20 \pm 58$ Mediumcts and $15 \pm 36$ Hardcts in the central $1 r_{c}$ radius region, and $60 \pm 24,75 \pm 28$ and $45 \pm 25$, respectively, in the surrounding 1-2 $r_{c}$ annulus. Thus, as suspected in GHE01a, there is an excess of Softcts in the cluster core, but the uncertainties in this extraction are large and no excess in the core of Mediumcts or Hardcts is required. For the detected MSPs with 
Softcts as listed in Table 1 (with mean value 6cts), the excess total Softcts $(240)$ could arise from an additional $\sim 40$ MSPs, all with $\mathrm{L}_{x} \gtrsim 10^{30} \mathrm{erg} \mathrm{s}^{-1}$. Since some of these soft sources could be BY Dras (coronal sources are typically soft) or CVs, as already noted (GHE01a), we regard 40 as an upper limit and estimate 20 (half) "unresolved" MSPs in the core.

Scaling by our known sample of 16 MSPs, we derive $40 \times$ $(16 / 7)=91$ MSPs as an upper limit on the total MSPs in the cluster. This upper limit agrees with the upper limit derived from consideration of WAVDETECT soft sources, and we adopt 90 as an approximate maximum number of MSPs in 47 Tuc with $\mathrm{L}_{x} \gtrsim 10^{30} \mathrm{erg} \mathrm{s}^{-1}$. Both of these estimates assume that no beaming of the MSPs in X-rays is occurring. (Note that thermal polar cap emission, while pulsed, is not beamed.) If there are more MSPs than this in 47 Tuc, they must have relatively hard spectra (since the above totals are scaled from the soft source counts) which could make them like the MSP in NGC 6397 (see below) for which radio emission is occulted much of the time. Alternatively, additional MSPs are beamed away from us in the X-ray, or of significantly lower x-ray luminosity on average. The softer color of the excess counts than our known MSP sample suggests that either the excess counts are composed largely of other sources (such as faint BY Dra binaries), or that there is a significant population of faint (in X-ray and radio) MSPs which would have cooler (and thus softer) polar caps.

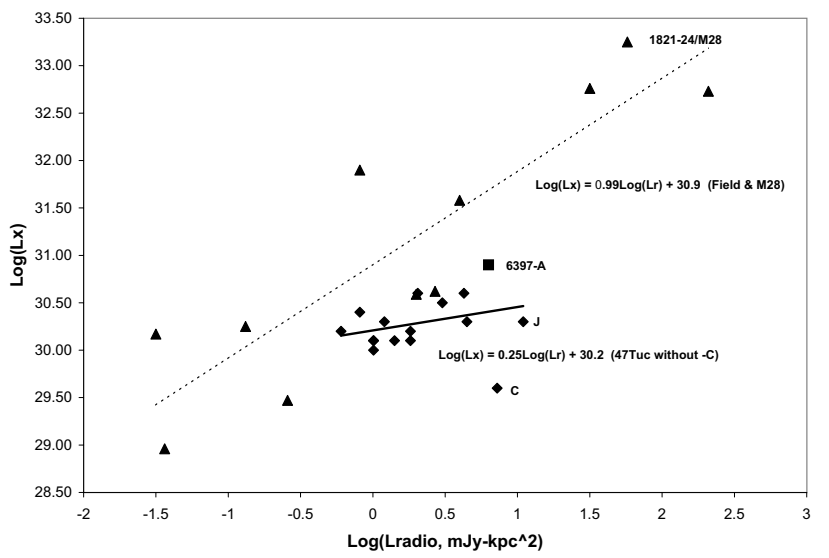

FIG. 7. $-\mathrm{L}_{x}$ vs. $\mathrm{L}_{\text {radio }}$ for MSPs in 47 Tuc (diamonds) and field (triangles). MSP in NGC 6397 (square) is labeled as is -J in 47 Tuc. Values for MSPs in field and M28 are from Table 2, where $\mathrm{L}_{\text {radio }}=S_{1400} d^{2}$. 47 Tuc MSP $S_{1400}$ values are from Camilo et al. (2000). Correlation line shown with 47 Tuc-C excluded (see text).

A significant number of additional ( $\gtrsim 15-70$ ) MSPs already detected in the Chandra data but not yet detected in the deep Parkes radio surveys is quite possible, given the relatively flat dependence of soft $\mathrm{x}$-ray luminosity, $\mathrm{L}_{x}$, on effective radio luminosity, $\mathrm{L}_{\text {radio }}=S_{1400} d^{2}$, where $S_{1400}$ is the flux density at $1400 \mathrm{MHz}$ and $d$ the MSP distance. This relation is shown in Figure 7 for 47 Tuc, NGC 6397, M28 and field MSPs. The 47 Tuc MSPs have a distinctly flatter $\mathrm{L}_{x}$ dependence on $\mathrm{L}_{\text {radio }}$, with logarithmic slope $0.00 \pm 0.23$ for all the 47 Tuc MSPs or $0.25 \pm 0.15$ with 47 Tuc-C excluded from the fit, as shown in Figure 7. The logarithmic slope is $0.99 \pm 0.17$ for the field MSPs (and M28), which is at least partly affected by sample selection bias. Thus it appears that in 47 Tuc MSPs that are fainter at radio wavelengths are not as much fainter in X-rays and should be more readily detected in Chandra data than in Parkes data. The ratio of 47 Tuc MSPs likely detected in x-rays ( 35-90) vs. radio (20) can constrain the relative beaming fractions since both samples are detected above the same $\mathrm{X}$-ray luminosity limit $\left(\mathrm{L}_{x}=10^{30} \mathrm{erg} \mathrm{s}^{-1}\right)$. If the $\mathrm{x}$-rays are not beamed, the implied radio beaming fraction is $\sim 0.2-0.5$.

We cannot (yet) isolate the candidate MSPs among the unresolved component, but as a possible aid to future MSP identifications, we report the positions and fluxes of the 19 Soft sources detected which are not identified with possible BY Dra or CV candidates. In Table 5 we give the source positions, detected counts, and approximate luminosities $\mathrm{L}_{x}$ (again, assuming for consistency a $\mathrm{kT}=0.2 \mathrm{keV} \mathrm{BB}$ spectrum in the $0.5-2.5 \mathrm{keV}$ band). These are all possible MSP candidates (though some BY Dras and a few CVs are likely included) and thus appropriate for use as trial positions in timing solutions for detected radio MSPs.

\section{COMPARISON WITH MSP IN NGC 6397}

We now compare the properties of the 47 Tuc MSPs with those derived for the single MSP in NGC 6397. This MSP, PSR J1740-5340 (D'Amico et al. 2001a), hereafter 6397-A, was recently located by a pulse timing solution (DPM01) and identified with an optical counterpart (Ferraro et al. 2001) previously identified with a main sequence binary or BY Dra system (TGE01). Chandra observations of NGC 6397 (GHE01b) detected 20 sources within $1^{\prime}$ of the cluster center, including the MSP. Because the Chandra sources included $8 \mathrm{CVs}$ firmly identified with HST optical counterparts displaying $\mathrm{H} \alpha$ emission (GHE01b), the Chandra-HST boresight could be measured to $\sim 0$.' $^{\prime} 01$ in RA and Dec. Thus the astrometric identification of the Chandra source U12 (GHE01b) with the apparent BY Dra binary WF4-1 found by TGE01 is secure (the ChandraHST positional offsets are given in Table 1, and the Chandraradio offsets are given in GHE01b). Since this binary has an optical photometric period 1.34 d (TGE01) which is consistent with that of 6397-A (D'Amico et al. 2001a), and is included in the $\sim 1^{\prime \prime}$ astrometric uncertainty of the HST guide star vs. radio timing reference frames, it was identified as the probable optical counterpart by Ferraro et al. (2001). In addition to the precise Chandra positional identification of the $1.34 \mathrm{~d}$ binary, we confirm the MSP identification by its X-ray temporal variability which was mentioned by GHE01b as being consistent with modulation at the binary period.

In Figure 8 we show the x-ray light curve of 6397-A (source U12) plotted against the binary phase as determined from the ephemeris of DPM01. The count rate increases smoothly by a factor of $\sim 2$ at phase $\sim 0.4$, or just before the MSP comes out of radio eclipse (which is centered at phase 0.25 ; phase 0 is the time of ascending node), removing any doubt that the MSP is the $\mathrm{x}$-ray counterpart. The fact that the flux is not zero during eclipse (though Chandra coverage only begins at phase $\sim 0.1$ ) suggests that the $\mathrm{x}$-ray source is extended and larger than the companion size, which has radius $\sim 1.3-1.8 R_{\odot}$ and nearly fills its Roche lobe (Burderi et al. 2002). Thus the X-ray emission is likely to be from the relativistic wind from the MSP interacting with the mass loss from the companion. Any thermal (BB) component from the MSP itself must be $\lesssim 0.5$ the total flux, as constrained by the flux increase out of eclipse. The hardness ratios during $\left(\mathrm{HR} 1=4.7_{-1.6}^{+5}, \mathrm{HR} 2=0.42 \pm 0.16\right)$ and outside eclipse $\left(\mathrm{HR} 1=3_{-0.9}^{+1.7}, \mathrm{HR} 2=0.57 \pm 0.2\right)$ remain the same within the errors, implying the modulation is not due to increased $N_{H}$. More sensitivity and phase coverage is needed to confirm and constrain the modulation. 
The x-ray counts detected in each of the Soft, Medium and Hard bands are given in Table 1, together with the $\dot{E}$ value from DPM01, which assumes that, given its relatively large projected offset of 0!6 $\left(\sim 11 r_{c}\right)$ from the cluster center, the cluster acceleration may be ignored. MSP 6397-A is clearly very hard, with hardness ratios HR1 $=3.6$ and HR2 $=0.5$. We do not plot it in Figure 2 since the very different absorption column $\left(N_{H}=1.0\right.$ $\times 10^{21} \mathrm{~cm}^{-2}$, or $\gtrsim 4 \times$ that for 47 Tuc) precludes direct comparison with the models plotted for 47 Tuc, but it is consistent with a PL with photon index $\sim 1.5$ or TB with $\mathrm{kT} \gtrsim 5 \mathrm{keV}$.

However we can compare the derived $\dot{E}$ and characteristic age, and their relation to $\mathrm{L}_{x}$, for this MSP with the 47 Tuc sample. In Figures 3 and 4 we plot 6397-A but do not include it with the linear fits which were derived for the 47 Tuc MSPs alone. It is clear that 6397-A is consistent with the 47 Tuc sample and correlations shown in both figures. Including it on the $\mathrm{L}_{x}$ vs. $\dot{E}$ correlation would give a combined (47 Tuc and NGC 6397) $\mathrm{L}_{x} \propto \dot{E}^{\beta}$ relation with $\beta=0.48 \pm 0.15$, even including the errors on all the points (despite the bias towards steepening the slope in doing so, as mentioned before). This is at first surprising, given the very different spectral shape and thus emission region, as well as (much) smaller spindown age, $\log (P / 2 \dot{P})=8.5$, for 6397-A. However the eclipsing MSP in 47 Tuc with the hardest spectrum, 47 Tuc-J, already hints that the $\mathrm{X}$-ray spectral shape is not critical to the $\mathrm{L}_{x}$ vs. $\dot{E}$ correlation, since $-\mathrm{J}$ does not depart from the correlation line. Thus there seems to be a relatively fixed $\mathrm{L}_{x}(0.5-2.5 \mathrm{keV})$ for both the 47 Tuc MSPs and 6397-A which emerges for a given spindown power $\dot{E}$ regardless of the emission mechanism. It appears that the conversion of $\dot{E}$ into thermal (BB) emission from the NS and emission probably tied to the relativistic pulsar wind are both fixed by the total polar cap current which, from the models of HM02 is in turn proportional to $\dot{E}^{1 / 2}$ (see §5).

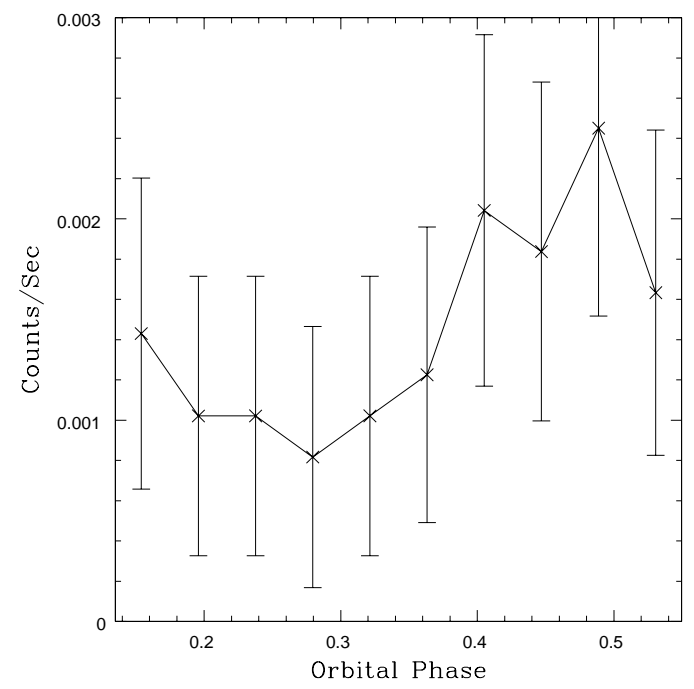

FIG. 8.- X-ray count rate $(0.5-4.5 \mathrm{keV})$ vs. orbital phase (from ephemeris of DPM01) for the MSP in NGC 6397, source U12 of GHE01b, observed for $49 \mathrm{ksec}$ beginning at UT $31.647 \mathrm{July}, 2000$. The source is persistently eclipsed in the radio at phases $0.05-0.45$. The $\mathrm{x}$-ray detection at this phase, and increase at phase $\gtrsim 0.4$, imply at least part of the $\mathrm{x}$-ray emission is extended.

Finally, we note that the large radial offset of 6397-A in the cluster, at radius $\sim 11 r_{c}$, is much larger than any of the detected, or plausible new candidate, MSPs in 47 Tuc. DPM01 and GHE01b noted it was likely ejected from the core, perhaps in a second exchange encounter since the MSP companion is not the expected He WD or low mass (ablated?) remnant of a secondary in an LMXB (as for all previously identified MSP companions) but rather is apparently just off the main sequence. GHE01b noted that 6397-A is approximately aligned with the central "line" of blue stragglers as well as several other of the Chandra sources - suggesting possible asymmetric ejection of binaries from the cluster core (possibly induced by cluster rotation; R. Spurzem, private communication). Since NGC 6397 is a post core collapse cluster with a power law cusp measured out to $\sim 60^{\prime \prime}$ (Lugger, Cohn and Grindlay 1995) that is expected to contain scattered binaries (and NSs), the MSP has surely been scattered.

Burderi et al. (2002) show that 6397-A could have evolved from an initial $\sim 14 \mathrm{~h}$ binary period to its present $34 \mathrm{~h}$ period, near the critical value where pressure from the MSP wind exceeds that of matter overflowing the Roche lobe (though intermittent accretion could resume; cf. §5). However the scattering encounter probably occurred within the past $\sim 10^{7} \mathrm{yr}$ since the central relaxation time is only $\sim 10^{5}$ yr (Harris 1996) and still $\lesssim 10^{6-7} \mathrm{yr}$ at the current offset position. An upper limit to the scattering time is $\sim 10^{8-9} \mathrm{y}$, the likely time since core collapse and also the likely MSP "age" (Figure 4a). The scattering timescale is thus shorter than the binary or nuclear evolution timescales leading to the present system, and the interaction must have altered the binary parameters. Most probably, the binary hardened in the encounter if the present secondary was the original donor. This makes it less likely that the secondary, now just inside its Roche lobe, could have filled its Roche lobe prior to the encounter and spun the MSP up in an LMXB phase. If mass transfer occurred only after the hardening induced by the encounter, there would not be enough time to spin up the MSP given the $\dot{m} \sim 10^{-10} M_{\odot} \mathrm{yr}^{-1}$ for the likely intermittent LMXB phases (Burderi et al. 2002) and the MSP "age".

If, however, the current secondary was exchanged into the binary displacing what was the original donor (probably a much lower mass HeWD, as in most other MSPs), there would not be the MSP formation-timescale problem. Even more interesting, this may help explain a puzzle pointed out by Orosz \& van Kerkwijk (2002) after this paper was submitted: the measured (DPM01) $\dot{E}$ for 6397-A would suggest significant photometric heating of the binary companion (by the MSP wind) though no visible heating modulation (only ellipsoidal variation) is observed. The "excess" $\dot{E}$ might be a factor of 2-3, depending on uncertain heat transport in the secondary. Orosz \& van Kerkwijk suggest the large $\dot{E}$ may be due to a $\dot{P}$ value dominated by a triple companion (easily tested by longer term MSP timing). If, instead, the present secondary has been exchanged for the original, and it is marginally Roche lobe filling (Burderi et al. 2002), then accretion (and LMXB) episodes could lead to spindown episodes of the MSP - at least for those encounters that are retrograde but possibly also for the very misaligned prograde case. Depending on uncertain crust-core coupling timescales, an increased $\dot{P}$ (negative; or positive for the nearly aligned prograde case) may persist for the MSP between intermittent accretion episodes. This enhanced $\dot{P}$ in an "old" MSP with low B field would not produce the same enhanced pulsar wind (and thus heating) as a nominal "young" (higher B field) MSP since $\dot{P}$ is now dominated by internal, rather than electromagnetic, rotation loss. The true age of 6397-A would thus be correspondingly older. Of course reduction of $\dot{E}$ would move 6397-A off the apparent correlation lines (e.g. Figs. 3, 4a) defined by the 47 Tuc MSPs, though if (only) a factor of $\sim 2-3$ reduction in 
$\dot{E}$ this might be compensated if only the thermal component of $\mathrm{L}_{x}$ (not yet isolated) were plotted.

A second example of a possible "double-exchange" MSP, with a probable main sequence secondary, has been discovered with the optical identification of the MSP 47 Tuc-W: Edmonds et al. (2002) report (after this paper was submitted) the identification of Chandra source W29 (cf. GHE01a) with a V=22.3 star in the central core $\left(\leqslant 0.2 \mathrm{r}_{c}\right.$ from the center) of 47 Tuc with optical modulation identical in both period (to within $0.5 \mathrm{~s}$ ) and phase (to within $1.2 \mathrm{~min}$ ) with the $3.2 \mathrm{~h}$ binary modulation of 47 Tuc-W. The x-ray luminosity and hardness ratios for the Chandra source (W29) are very similar to those for 6397-A. Although this is a certain MSP identification, its one-time $(\sim 4 \mathrm{~h})$ radio detection (Camilo et al. 2000) precludes measurement of its $\dot{P}$ and thus $\dot{E}$ value (possibly enhanced?) so that it cannot be included (now) in our analysis of the full radio MSP sample in 47 Tuc. Its identification does show, however, that our estimates for the total MSP sample in 47 Tuc as derived from the soft source distribution are likely to be lower limits. Indeed, Edmonds et al. (2002) also find in the core of 47 Tuc a second possible MSP with main sequence companion as the optical counterpart of Chandra source W34, with orbital period $97.45 \mathrm{~m}$ (not matched to any of the radio MSP sample).

\section{DISCUSSION}

One of the key results of this study is that MSPs in the globular clusters 47 Tuc and NGC 6397 may have a less efficient conversion of rotational spindown energy $(\dot{E})$ into soft x-rays $\left(\mathrm{L}_{x}\right)$ than most field MSPs, even those with correspondingly low values of their magnetic field at the light cylinder. For $\mathrm{L}_{x} \propto \dot{E}^{\beta}$, the 47 Tuc-NGC 6397 samples are fit by $\beta=0.5 \pm 0.15$ whereas the field and M28 sample are consistent with the value $\beta=1$ found for non-recycled pulsars. The 4 field MSPs with largest $\dot{E}$ and $B_{\text {lc }}$ (cf. Figs. 3 and 5) show correlations with $\mathrm{L}_{x}$ which extrapolate to the low $\dot{E}$ and $B_{\mathrm{lc}}$ sample, whereas the correlation shown by the 47 Tuc-NGC 6397 MSPs does not extrapolate to higher $\dot{E}$ and $B_{\text {lc }}$ systems. It is possible that the difference is just due to the 4 MSPs with $B_{\mathrm{lc}} \gtrsim 10^{5.5} \mathrm{G}$ being dominated by nonthermal beamed emission (e.g. since B1821-24 has no binary companion, its hard spectrum is not from a MSP wind interacting with a companion, as plausibly is the case for 47 Tuc-J and 6397-A). However, then the large deviations from the 47 Tuc correlation of the field MSPs at lower $B_{\mathrm{lc}}$ must be explained as systematic errors in distance, which is unlikely for at least two of them. Perhaps more convincing, the $\mathrm{L}_{x} / \dot{E}$ vs. $\tau$ relations for the field vs. 47 Tuc MSPs (Fig. 4b) show significant deviations for the low $B_{\mathrm{lc}}$ systems. What could be different about the MSPs in these two globulars vs. field MSPs?

The polar cap (PC) heating models of HM02 can predict the observed $\mathrm{L}_{x}-E^{\beta}$ relation, with $\beta \sim 0.5$, for MSPs with spindown ages $\tau=P / 2 \dot{P} \gtrsim 10^{8}$ yr that are no longer able to produce pairs by curvature radiation (CR) $\gamma$-rays (which convert to pairs in the pulsar magnetic field) but instead by $\gamma$ 's from inverse Compton scattering (ICS) of thermal photons from the NS surface. >From the numerical models for PC heating by returning positrons, with total heating luminosity $\mathrm{L}_{+}$for the ICS regime and the resulting prediction for $\mathrm{L}_{+} / \dot{E}$ vs. $\tau$ (HM02, Fig. 8 b), we find the approximate empirical relations $\mathrm{L}_{+} / \dot{E} \propto \tau^{\alpha} P^{2 \alpha}$ which yields $\mathrm{L}_{+} \propto \dot{E}^{1-\alpha}$ and $\mathrm{L}_{+} \propto \tau^{\alpha-1} / P^{2(1-\alpha)}$ using $\tau \propto$ $1 / \dot{E} P^{2}$. For MSPs with $\tau \lesssim 1-2 \times 10^{9}$ yr (beyond which the expected $\mathrm{L}_{+} / \dot{E}$ decreases as the MSP approaches the pair deathline), this gives $\alpha \sim 0.4-0.5$, or $\mathrm{L}_{+} \propto E^{\beta}$, or $\beta=1-\alpha=0.5-$
0.6. The model predicts (for $P=5 \mathrm{~ms}$ and PC temperature $\mathrm{T}=3$ $\left.\times 10^{6} \mathrm{~K}\right) \log \left(\mathrm{L}_{x} / \dot{E}\right) \sim-4.5+0.4 \log \left(\tau / 10^{9} \mathrm{yr}\right)$ and so is consistent with the approximate trend from 6397-A to the apparently youngest 47 Tuc MSPs. However, several key differences are evident: (i) the 47 Tuc MSPs (and 6397-A) show an unbroken power law dependence on age, with $\mathrm{L}_{x} \propto L_{+} \propto \tau^{-0.3}$ (Fig. 4a), well beyond the expected steepening (HM02, Fig. 9b) at $\tau \sim 10^{9} \mathrm{yr}$, (ii) the $\mathrm{L}_{x} / E$ relation (Fig. $4 \mathrm{~b}$ ) likewise continues to increase beyond the expected flattening at similar $\tau \sim 10^{9} \mathrm{yr}$ (HM02, Fig. 8b). In contrast, the field MSPs in Figures 4a,b are more consistent with the HM02 models, having negative or flat slopes, respectively, at $\tau \gtrsim 10^{9} \mathrm{yr}$. However, the factor of $\sim 3-10 \times$ excess of $\mathrm{L}_{x} / \dot{E}$ over those for the 47 Tuc MSPs and the ICS models also suggests these systems have an additional source of $\mathrm{L}_{x}$ : probably non-thermal emission. It is interesting that accounting in Figure $4 \mathrm{~b}$ for the approximate dependence on pulsar period, $\mathrm{L}_{x} / \dot{E} \propto P^{2 \alpha}$ (derived from a fit to Fig. $8 \mathrm{~b}$ of HM02), makes the field MSPs have a (slightly) more negative slope $(-0.16 \pm 0.23$ vs. $-0.06 \pm 0.21)$, as expected from HM02 for their age, whereas the 47 Tuc MSPs have a slightly more positive logarithmic slope $(0.59 \pm 0.10$ vs. $0.53 \pm 0.13)$. Finally, (iii) the small effective $\mathrm{BB}$ emission areas $\left(\mathrm{r}_{x} \lesssim 0.14-0.56 \mathrm{~km}\right.$ for dipole polar caps; smaller still (each) for multipole caps) are significantly smaller than the effective radius of the annular polar cap emission region predicted (HM02 Fig. 7).

We suggest that these differences may point to altered surface magnetic field configurations for the 47 Tuc (and 6397-A) MSPs to enable such efficient PC heating at large age. If their surface magnetic fields have significant multipole components, the effective B-field curvature is greater and the pair production front moves closer to the NS yielding more efficient positron return to heat the polar caps. The magnetic field footprint on the NS becomes smaller, giving the small emission areas. If the non-thermal radio emission is produced, as in most models, from near the light cylinder where the field is dominated by the dipole component, then its properties are not significantly altered and the radio pulse profiles are otherwise similar to field MSPs (as observed; Camilo et al. 2000). Clearly this multipole field hypothesis must be tested with models (only dipole fields have been considered for pulsar emission and PC heating e.g. HM02).

Another possibility is that the NSs in the 47 Tuc MSPs (and 6397-A) are systematically more massive or compact than those in the field MSPs. The models of Harding, Muslimov, \& Zhang (2002) show that the ICS regime becomes longer lived (extends to lower $\dot{P}$ values) for more massive or more compact NSs. Although our radial distribution results (\$3.2) imply MSP masses (total) in the range $\sim 1.1-1.4 M_{\odot}$, suggesting the more compact NS interpretation may be preferred, more massive NSs are allowed if in fact the MSP distribution is "puffed up" by binary scattering.

The hard spectra in a few of these "thermal" MSPs (47 Tuc$\mathrm{J}$ and 6397-A) must have a different origin. In 6397-A, the possible modulation phased with the radio eclipse of the hard source suggests the hard source is due to shocked gas lifted from the binary companion by the MSP wind pressure (Burderi et al. 2002). If the hard spectrum is a power law (thermal bremsstrahlung is ruled out by the $\delta \mathrm{DM}$ constraint [\$2.1] unless the emission is strongly aspherical), and the shocked wind density and thus emission measure is proportional to the underlying MSP thermal luminosity, then the approximate agreement of these hard spectrum, but low $\mathrm{L}_{x}$, systems with the $\beta=0.5$ 
law exhibited by the soft (thermal) MSPs would imply scaling of the shocked gas emission with the thermal (NS) emission. As mentioned above, this might be expected if both the MSP wind and PC heating are proportional to the PC current.

What is special about globular cluster MSPs to drive them towards altered $B_{\text {surf }}$ (and thus $B_{\mathrm{lc}}$ ) values or configurations or possibly larger NS mass (or compactness)? Unlike MSPs in the field, those in dense cluster cores have a possibility of being driven back into contact and an accretion phase, as a re-cycled LMXB (from a MSP)! Renewed accretion (and the 6397-A companion is presently close to filling its Roche lobe; cf. DPM01) would likely continue the B-field burial process thought to be responsible (e.g. Romani 1990) for field decay from the $\gtrsim 10^{11} \mathrm{G}$ fields at NS birth to the $\lesssim 10^{9} \mathrm{G}$ values typical of MSPs. This could lead, in turn, to altered Bfield configuration (multipole field components), particularly for a secondary re-exchanged into an MSP (with random spinorbital encounter angular momentum). It would also systematically increase the NS mass beyond that for field MSPs. The 47 Tuc MSPs have spent their lifetime $\tau \gtrsim 1 \mathrm{Gyr}$ in a dense ( $n \sim 10^{5} \mathrm{pc}^{-3}$ ) cluster core, where they undergo scattering interactions with both single stars and other binaries. Such scattering causes the binding energy $x$ of a hard binary to secularly increase ("hard binaries harden") at a rate $\dot{x} \sim 4 m \sigma^{2} n p_{0}^{2} \sigma$ (Goodman \& Hut 1993), where $m$ is the single star mass, $\sigma$ is the one-dimensional velocity dispersion, and $p_{0}=\mathrm{Gm} / \sigma^{2}$ is the impact parameter for a $90^{\circ}$ deflection $(\sim 10 \mathrm{AU}$ in the core of 47 Tuc). Thus, the binding energy of a typical hard binary increases by of order $m \sigma^{2}$ per core relaxation time $\left(\sim 10^{8} \mathrm{yr}\right.$ for 47 Tuc). Over the $\gtrsim 1$ Gyr lifetime of the MSP binary system, the total relative binding energy change is $\dot{x} \tau / x \sim 1 \%$. Thus, while angular momentum transfer to the secondary acts to detach the binary, scattering events tend to drive it back toward contact.

A complication in this picture is that since MSPs are extremely hard binaries $\left(x \sim 1000 m \sigma^{2}\right), x$ is adiabatically invariant in weak scattering events where the distance of closest approach much exceeds the binary separation (e.g. Heggie \& Hut 1993). Thus, the secular increase in $x$ is largely the result of infrequent strong scattering events, which are likely to result in an ejection of the binary from the cluster core (e.g. 6397-A) or even the cluster. Other unresolved issues include the effects of binary-binary scattering, which probably "bump" the MSPs back into contact more effectively than does binarysingle scattering, and orbital eccentricity evolution during scattering events, which will also tend to drive some binaries towards contact. Nevertheless, it appears plausible that a typical old ( $\tau \gtrsim 1 \mathrm{Gyr}$ ) MSP in a dense cluster core might undergo one or more MSP-LMXB recurrence cycles. Thus a few percent of the MSPs at any one time may be in (or near) this recurrent LMXB phase. This might explain the puzzling luminous qLMXBs X5 and X7 (GHE01a and Heinke et al. 2002) in 47 Tuc: they could be recently $\left(\sim 10^{5-6} \mathrm{yr}\right)$ revived qLMXBs, in which their underlying MSP nature is hidden by a heated (by the MSP wind) outflow from the disk and Roche lobe of the probable main sequence secondaries (at least for X5, which may then be similar to 6397-A and 47 Tuc-W; cf. §4).

The (possibly) younger system in NGC 6397 has the advantages of having been recently scattered out of a still higher density $\left(\sim 10^{6} \mathrm{pc}^{-3}\right)$ core collapsed cluster core and possibly having exchanged its companion. Either or both would likely have restored an accretion phase. Thus the MSP need not be just "born," as suggested by Ferraro et al. (2001); it may instead have just been reborn. In contrast, the MSP in M28 is both $\sim 10 \times$ younger, single and in a lower density core and so is unlikely to have gone through a renewed accretion phase. This MSP-LMXB recycling scenario would imply: (i) a population of MSPs with main sequence or red straggler secondaries (either primordial or re-exchanged) like 6397-A which may be largely eclipsed (in radio) by mass loss and dominated by hard shocked gas emission, (ii) a strong dependence for this multiply-recycled population to occur primarily in clusters with largest central mass density $\rho_{o}=\mathrm{n}_{o} \mathrm{~m}$ and core radius $\mathrm{r}_{c}$, and thus total interaction rate $\left(\Gamma \propto \rho_{o}^{1.5} r_{c}^{2}\right)$ such as Terzan 5, and (iii) that multiple MSP-LMXB phases may be more likely, thus yielding $B_{\text {surf }}$ burial and altered $B_{\mathrm{lc}}$ and suppressed magnetospheric emission, in MSPs with main sequence companions. MSPs with either main sequence or slightly evolved secondaries (e.g. 47 Tuc-W or 6397-A; cf. § 4) are also most likely to contain re-exchanged secondaries which may have anomalous $\dot{P}$ values (as might 6397-A) from the braking torque supplied by a mis-aligned crust-core spin coupling due to the renewed accretion phase(s) from the re-exchanged secondary. Even an original secondary, if driven back into contact or if marginally filling its Roche lobe, could give rise to enhanced $\dot{P}$ values: when accretion stops, the crust is spinning faster than the core, which then exerts a braking torque on the crust (and B-field) of the MSP. The timescale for equilibrium to be established is uncertain but likely to be relevant for MSPs with companions closest to filling their Roche lobes (hence the main sequence or subgiant systems) which have most recently accreted. These main sequence systems, which likely evolve into the short period ( $\sim 1.5-5.5 \mathrm{~h})$ group identified by Camilo et al. (2000), also have longer lifetimes to undergo binary scattering than those spun-up from sub-giant companions and now in the longer period ( 0.4-2.4d) group with He-WD companions. However this lifetime is partly offset by the larger binary cross sections for the He-WD group, so both are expected to undergo scattering in dense cores. The generally larger orbital eccentricities for He-WD secondary MSPs in globulars vs. those in the disk has previously been noted (Phinney 1992) as evidence for interaction of these MSPs with cluster stars. The large eccentricity of 47 Tuc-H (FCL01) suggests it has undergone such an encounter.

\section{CONCLUSIONS}

In this paper we have examined the $\mathrm{x}$-ray properties of the full MSP populations in 47 Tuc and NGC 6397 localized via radio timing. The large sample of MSPs resolved by Chandra in 47 Tuc, and the single system in NGC 6397, allow a number of conclusions to be drawn:

- The MSPs in 47 Tuc and NGC 6397 are surprisingly uniform in $\mathrm{L}_{x}$ despite a much larger variation in $\dot{E}$. The $\mathrm{L}_{x}-\dot{E}$ correlation appears to be significantly flatter than for a (smaller) sample of MSPs in the field as well as the isolated (and young) MSP in M28. The correspondingly flatter $\mathrm{L}_{x}-B_{\mathrm{lc}}$ correlation suggests that the 47 Tuc (and NGC 6397) MSPs may have $B_{\text {surf }}$ and thus $B_{\text {lc }}$ configurations systematically different (e.g. multipole components) from field MSPs and which prolong thermal and suppress magnetospheric $\mathrm{x}$-ray emission.

- The MSPs in 47 Tuc are dominated by thermal emission sources, consistent with BB emission from the pul- 
sar polar caps. At least one (47 Tuc-J) must also have significant non-thermal emission, which may arise from the pulsar wind interacting with the companion (see below).

- The radial distribution of MSPs in 47 Tuc is consistent with the predictions of a multi-mass King model for a distribution of MSPs with mass ratio $q \sim 2$ times the typical cluster stellar mass in the core, i.e. a MSP system mass of $\sim 1.4 M_{\odot}$.

- From the distribution of Chandra sources with similarly soft X-ray colors, as well as the total counts detected in Soft and Medium bands vs. cluster radius, the number of MSP candidates with $\mathrm{L}_{x} \gtrsim 1 \times 10^{30} \mathrm{erg} \mathrm{s}^{-1}$ is estimated to be $\sim 35-90$, allowing for the plausible contamination of BY Dra and soft CV systems and incompleteness of the detection for faint sources near the cluster center. The relative number of MSPs detected in the complete soft $\mathrm{x}$-ray vs. radio samples suggests the radio emission is beamed with beaming factor $\sim 0.2$ 0.5 (times any soft $\mathrm{x}$-ray beaming, which is presumably isotropic).

- A larger population of still fainter MSPs $\left(L_{X}<10^{3} 0\right.$ $\mathrm{erg} \mathrm{s}^{-1}$ ) could be detected in $\mathrm{x}$-rays than in the radio, given the relatively flat $\mathrm{L}_{x}-\mathrm{L}_{\text {radio }}$ correlation seen for 47 Tuc MSPs. If the excess of Soft vs. Medium band counts in the cluster core is due to fainter MSPs, they must have relatively lower luminosity and lower temperatures than the identified MSPs.

- The one (binary) MSP system identified in the corecollapsed globular NGC 6397 is consistent with the $\mathrm{L}_{x}-$ $\dot{E}$ relation of the 47 Tuc sample despite its very different spectrum, which must be dominated by non-thermal or bremsstrahlung emission. Its harder x-ray emission must (largely) arise from an extended source, possibly resulting from interaction of the relativistic wind from the MSP with mass loss from its companion star. The fact that it is still consistent with the $\mathrm{L}_{x} \propto \dot{E}^{1 / 2}$ relation found for the thermal MSPs in 47 Tuc suggests that both the pulsar wind luminosity and polar cap heating are governed by the total polar cap current $\left(\propto \dot{E}^{1 / 2}\right.$; cf. HM02). If $\dot{P}$ is enhanced by renewed accretion or second exchanges, the electromagnetic spindown rate and corresponding reduced $\dot{E}$ may correlate with the thermal component of $\mathrm{L}_{x}$ (to be measured) as for 47 Tuc.

- The enhanced thermal luminosity, small emission areas and extension to larger MSP ages, for the 47 Tuc MSPs vs. the HM02 models suggests the surface magnetic field might be multipole (dipole at the light cylinder radius and probable radio emission region), possibly by field perturbations from repeated accretion events due to encounters or re-exchanges of the secondaries of these MSPs in dense cluster cores. Alternatively, the extended pair heating (and thermal x-rays) could arise (Harding, Muslimov, \& Zhang 2002) if the NSs in these MSPs are more massive or (given their radial distribution) more compact. If re-exchanged secondaries are present (as perhaps for $6397-$ A and 47 Tuc-W), apparent $\dot{P}$ and thus $\dot{E}$ values may be enhanced by core-crust coupling drag effects over their purely electromagnetic spindown values which give rise to heating their companions. Some evidence for a similar "excess" $\dot{E}$ value is available for 47 Tuc-W (Edmonds et al. 2002), but as with 6397-A these estimates are subject to uncertain heating efficiencies and transport effects (which might heat the backside of the tidally-locked secondary).

- NGC 6397 appears to be deficient in its total MSP population per unit mass compared with 47 Tuc (GHE01b), if most cluster MSPs are like the 47 Tuc sample and dominated by soft thermal sources. Only 3-5 such objects are found in the sample of 20 Chandra sources detected, with $\mathrm{L}_{x}$ limits a factor of 2 lower than in 47 Tuc (GHE01b). Most of the other Chandra sources in NGC 6397 with comparable x-ray colors (or actual spectra) are identified with CVs (optically; cf. GHE01b and references therein) and thus not likely to be additional MSPs. However it is possible that a few of the main sequence binaries, or BY Dra candidates (TGE01) could also harbor MSP primaries as is the case for 6397A.

- Conversely, the very large possible BY Dra population in 47 Tuc (up to $\sim 10^{3}$ are possible; R. Gilliland, private communication) may contain a significant sample of MSPs as may have occurred in 6397-A: those that have likely had a cluster main sequence star exchanged into the binary, displacing the probable degenerate secondary which had evolved from the original mass donor to spin up the MSP. If the 6397-A system with such a secondary (now evolved or bloated; cf. § 4) is typical, and mass loss is more readily driven off its near Roche-lobe filling secondary by the MSP wind pressure (Burderi et al. 2002), then these systems will likely be dominated by shocked gas and appear hard. Since these main sequence secondary MSPs will typically have larger MSP heating (given their larger secondaries) and thus mass loss, they may be largely eclipsed until their secondaries have been ablated to become the very low mass group $\left(\sim 0.02 M_{\odot}\right)$ found (Camilo et al. 2000 ) in the 47 Tuc MSPs. Thus the total MSP sample in 47 Tuc would include faint hard sources as well as the soft, thermal-dominated, systems and the total MSP population would exceed the $\sim 35-90$ estimated from the soft source population alone.

We shall obtain a very deep (300ksec) cycle 3 Chandra observation of 47 Tuc to probe the complete MSP population as well as to better constrain and measure x-ray colors and actual spectra to test emission models. The hard spectra MSPs (e.g. 47 Tuc-J) may be variable (as is 6397-A), and hard components should be detectable in other eclipsing MSPs (e.g. 47 Tuc-O). The interlocking puzzles of compact binary populations (e.g. MSPs vs. BY Dra systems) and compact object populations (MSPs vs. CVs, as measures of NS vs. WD populations) in globular clusters offer new insights into the stellar and dynamical evolution of these oldest stellar systems in the Galaxy.

We thank Paulo Freire and Michael Kramer for help with and discussions regarding the radio data, Ron Gilliland for help with the HST data, Werner Becker for comments on the paper, Alice Harding for comments on PC models, Fred Lamb for comments on core-crust coupling effects and the referee Dick 
Manchester for suggestions. This work was supported in part by NASA grants GO0-1098A and HST-AR-09199.01-A (JG) and SAO grant GO1-2063X (FC).

\section{REFERENCES}

Albrow, M., Gilliland, R. L., Brown, T. M., Edmonds, P. D., Guhathakurta, P., \& Sarajedini, A. 2001, ApJ, 559, 1060

Aldcroft, T. L., Karovska, M., Cresitello-Ditmar, M. L., Cameron, R. A., \& Markevitch, M. L. 2000, Proc. SPIE, 4012, 650

Becker, W., \& Trümper, J. 1999, A\&A, 341, 803 (BT99)

Becker, W., Trümper, J., Lommen, A. N., \& Backer, D. C. 2000, ApJ, 545, 1015

Bhattacharya, D., \& van den Heuvel, E. P. J. 1991, Phys. Rep., 203, 1

Burderi, L., D’Antona, F. \& Burgay, M. 2002, ApJ, 574, 325

Camilo, F., Lorimer, D. R., Freire, P., Lyne, A. G., \& Manchester, R. N. 2000, ApJ, 535, 975

Clark, G. W. 1975, ApJ, 199, L143

D’Amico, N., Lyne, A. G., Manchester, R. N., Possenti, A., \& Camilo, F. 2001a, ApJ, 548, L171

D’Amico, N., Possenti, A., Manchester, R. N., Sarkissian, J., Lyne, A. G., \& Camilo, F. 2001b, ApJ, 561, L89 (DPM01)

Dobrzycki, A., Ebeling, H., Glotfelty, K., Freeman, P., Damiani, F., Elvis, M., \& Calderwood, T. 1999, CHANDRA Detect 1.1 User Guide

Edmonds, P. D., Gilliland, R. L., Heinke, C. O., Grindlay, J. E., \& Camilo, F. 2001, ApJ, 557, L57

Edmonds, P. D., Gilliland, R. L., Camilo, F., Heinke, C. O., \& Grindlay, J. E. 2002, ApJ, in press

Ferraro, F. R., Possenti, A., D’Amico, N., \& Sabbi, E. 2001, ApJ, 561, L93

Freire, P. C. 2001, Ph.D. thesis, Univ. Manchester

Freire, P. C., Camilo, F., Lorimer, D. R., Lyne, A. G., Manchester, R. N., \& D'Amico, N. 2001a, MNRAS, 326, 901 (FCL01)

Freire, P. C., Kramer, M., Lyne, A. G., Camilo, F., Manchester, R. N., \& D’Amico, N. 2001b, ApJ, 557, L105

Giacconi, R., et al. 2000, ApJ, 551, 624

Goodman, J. \& Hut, P. 1993, ApJ, 403, 271

Grindlay, J. E., Heinke, C. O., Edmonds, P. D., \& Murray, S. S. 2001a, Science, 292, 2292 (GHE01a)

Grindlay, J. E., Heinke, C. O., Edmonds, P. D., Murray, S. S., \& Cool, A. M. 2001b, ApJ, 563, L53 (GHE01b)

Grindlay, J. E., Hertz, P., Steiner, J. E., Murray, S. S., and Lightman, A. P. 1984, ApJ, 282, L13

Harding, A. K. \& Muslimov, A. G. 2002, ApJ, 568, 862 (HM02)

Harding, A. K., Muslimov, A. G. \& Zhang, B. 2002, astro-ph/0205077

Harris, W.E. 1996, AJ, 112, 1487

Heggie, D. C. \& Hut, P. 1993, ApJS, 85, 347

Heinke, C. O., Grindlay, J. E., Lloyd, D. A., \& Edmonds, P. D. 2002, ApJ, submitted
Howell, J. H., Guhathakurta, P., \& Gilliland, R. L. 2000, PASP, 112, 1200

Jerius, D., Donnelly, R. H., Tibbetts, M. S., Edgar, R. J., Gaetz, T. J., Schwartz, D. A., van Speybroeck, L. P., \& Zhao, P. 2000, Proc. SPIE, 4012, 17

Jernigan, J. G. \& Clark, G. W. 1979, ApJ, 231, L125

Lange, C., Camilo, F., Wex, N., Kramer, M., Backer, D. C., Lyne, A. G., \& Doroshenko, O. 2001, MNRAS, 326, 274

Lightman, A. P., Hertz, P., \& Grindlay, J. E. 1980, ApJ, 241, 367

Lommen, A. N., Zepka, A., Backer, D. C., McLaughlin, M., Cordes, J. M., Arzoumanian, Z., \& Xilouris, K. 2000, ApJ, 545, 1007

Lugger, P. M., Cohn, H. N., \& Grindlay, J. E. 1995, ApJ, 439, 191

Mardling, R. 1995, ApJ, 450, 732

Meylan, G. 1988, A\&A, 191, 215

Meylan, G., \& Mayor, M. 1986, A\&A, 166, 122

Murphy, B. W., Moore, C. A., Trotter, T. E., Cohn, H. N., \& Lugger, P. M. 1998, BAAS, 193, 60.01

Navarro, J., de Bruyn, G., Frail, D., Kulkarni, S. R., \& Lyne, A. G. 1995, ApJ, 455, L55

Orosz, J.A. \& van Kerkwijk, M.H. 2002, A\&A, submitted

Phinney, E. S. 1992, Phil. Trans. Roy. Soc. A, 341, 39

Possenti, A., Cerutti, R., Colpi, M., \& Mereghetti, S. 2002, A\&A, 387, 993

Rasio, F. A., Pfahl, E. D., \& Rappaport, S. 2000, ApJ, 532, L47

Romani, R. 1990, Nature, 347, 741

Takahashi, M. et al. 2001, ApJ, 554, 316

Taylor, J. H. \& Cordes, J. M. 1993, ApJ, 411, 674

Taylor, J. H., Manchester, R. N., Lyne, A. G., \& Camilo, F. 1995, unpublished (available at http://pulsar.princeton.edu/pulsar/catalog.shtml)

Taylor, J. M., Grindlay, J. E., Edmonds, P. D., \& Cool, A. M. 2001, ApJ, 553, 169 (TGE01)

Toscano, M., Bailes, M., Manchester, R., \& Sandhu, J. 1998, ApJ, 506, 863

Toscano, M., Britton, M. C., Manchester, R. N., Bailes, M., Sandhu, J. S., Kulkarni, S. R., \& Anderson, S. B. 1999a, ApJ, 523, L171

Toscano, M., Sandhu, J. S., Bailes, M., Manchester, R. N., Britton, M. C., Kulkarni, S. R., Anderson, S. B., \& Stappers, B. W. 1999b, MNRAS, 307, 925

van Straten, W., Bailes, M., Britton, M., Kulkarni, S. R., Anderson, S. B., Manchester, R. N., \& Sarkissian, J. 2001, Nature, 412, 158

Wijnands, R., \& van der Klis, M. 1998, Nature, 394, 344

Yi, I., \& Grindlay, J. E. 1998, ApJ, 505, 828

Zavlin, V. E., Pavlov, G. G., Sanwal, D., Manchester, R. N., Trümper, J., Halpern, J. P. and Becker, W. 2002 ApJ, 569, 894

Zoccali, M., et al. 2001, ApJ, 553, 733 
TABLE 1

CHANDRA RESUlts ON MSPS IN 47 TUC AND NGC 6397

\begin{tabular}{cccccccc}
\hline \hline MSP & $\begin{array}{c}\Delta \mathrm{RA}^{\mathrm{a}} \\
(\operatorname{arcsec})\end{array}$ & $\begin{array}{c}\Delta \text { Dec }^{\mathrm{a}} \\
(\operatorname{arcsec})\end{array}$ & $\begin{array}{c}\text { Softcts }^{\mathrm{b}} \\
(0.2-1 \mathrm{keV})\end{array}$ & $\begin{array}{c}\text { Mediumcts }^{\mathrm{b}} \\
(1-2 \mathrm{keV})\end{array}$ & $\begin{array}{c}\text { Hardcts }^{\mathrm{b}} \\
(2-8 \mathrm{keV})\end{array}$ & $\begin{array}{c}\log \left(\mathrm{L}_{x}\right)^{\mathrm{c}} \\
(0.5-2.5 \mathrm{keV})\end{array}$ & ${\log (\dot{E})^{\mathrm{d}}}$ \\
\hline 47 Tuc-C & $\ldots$ & $\ldots$ & 1 & 0 & 0 & 29.6 & 33.2 \\
47 Tuc-D & -0.17 & 0.19 & 5 & 7 & 1 & 30.3 & 33.7 \\
47 Tuc-E & 0.01 & -0.07 & 11 & 13 & 0 & 30.6 & 34.5 \\
47 Tuc-F & 0.32 & 0.06 & 10 & 9 & 1 & 30.5 & 34.4 \\
47 Tuc-G & 0.05 & 0.03 & 4 & 4 & 0 & 30.1 & 33.7 \\
47 Tuc-H & 0.03 & 0.01 & 5 & 4 & 0 & 30.1 & 33.2 \\
47 Tuc-I & 0.16 & 0.01 & 5 & 5 & 1 & 30.2 & 33.9 \\
47 Tuc-J & -0.03 & -0.07 & 3 & 5 & 5 & 30.3 & 34.4 \\
47 Tuc-L & $\ldots$ & $\ldots$ & $12(10)$ & 5 & $6(0)$ & 30.4 & 34.2 \\
47 Tuc-M & 0.36 & -0.01 & 2 & 3 & 0 & 30.1 & 33.5 \\
47 Tuc-N & 0.01 & 0.10 & 5 & 6 & 0 & 30.2 & 34.1 \\
47 Tuc-O & 0.10 & 0.19 & 17 & 11 & 0 & 30.6 & 34.4 \\
47 Tuc-Q & $\ldots$ & $\ldots$ & 5 & 3 & 0 & 30.1 & 34.3 \\
47 Tuc-S & -0.26 & -0.39 & 5 & 4 & 1 & 30.2 & $\ldots$ \\
47 Tuc-T & $\ldots$ & $\ldots$ & 4 & 3 & 0 & 30.0 & 33.8 \\
47 Tuc-U & 0.04 & -0.06 & 4 & 7 & 1 & 30.3 & 34.2 \\
6397-A & -0.05 & 0.09 & 11 & 40 & 20 & 30.9 & 35.1 \\
\hline
\end{tabular}

a $\triangle \mathrm{RA}$ and $\triangle \mathrm{Dec}$ offsets are Chandra WAVDETECT minus precise MSP radio (timing) position (47 Tuc) and Chandra WAVDETECT minus HST position (NGC 6397). The close pair 47 Tuc-F, -S are marginally resolved (see text) and thus have larger offsets; offsets are not listed for $-\mathrm{L},-\mathrm{Q},-\mathrm{T}$ and $-\mathrm{C}$, without WAVDETECT positions due to crowding or limited counts (see Fig. 1).

${ }^{b}$ Counts in each band derived from $1^{\prime \prime}$ aperture on radio MSP position. MSPs 47 Tuc-G and -I are indistinguishable; counts are tentatively divided based on respective $\dot{E}$ values. MSPs 47 Tuc-F and $-\mathrm{S}$ detected combined with WAVDETECT; counts estimated separately (see text). 47 Tuc-L suffers severe crowding; values in parentheses indicate best estimates removing other source contamination.

${ }^{\mathrm{c}} \mathrm{L}_{x}$ values (in erg s${ }^{-1}$ ) are computed (PIMMS) for an assumed blackbody spectrum with $\mathrm{kT}=0.22 \mathrm{keV}$ (see text) and include exposure correction factors of 2.5, 1.4 and 1.9 for 47 Tuc-C, -J, and -M, respectively (all other MSPs detected with uniform exposure factor 1.0). Approximate errors on $\log \mathrm{L}_{x}$ are \pm 0.3 for 47 Tuc-C and \pm 0.2 for the others.

${ }^{\mathrm{d}} \dot{E}$ values (in erg s${ }^{-1}$ ) are derived for the 47 Tuc MSPs assuming the MSPs are at 3D radii appropriate to their $\delta \mathrm{DM}$, determined using a constant gas density in the cluster (Freire et al. 2001b), and a King model for the cluster potential with central velocity dispersion $11.6 \mathrm{~km} \mathrm{~s}^{-1}$. $\dot{E}$ value for 47 Tuc-S is indeterminate. $\dot{E}$ for the MSP 6397-A, at relatively large radial offset in NGC 6397, is derived assuming the cluster acceleration contribution is small. 
TABLE 2

PARAMETERS For MSPS PREVIOUSLY Detected IN X-RAYS

\begin{tabular}{lcccccc}
\hline \hline \multicolumn{1}{c}{ MSP } & $\begin{array}{c}P \\
(\mathrm{~ms})\end{array}$ & $\begin{array}{c}\dot{P_{i}} \\
\left(\times 10^{-20}\right)\end{array}$ & $\begin{array}{c}d \\
(\mathrm{kpc})\end{array}$ & $\begin{array}{c}S_{1400} \\
(\mathrm{mJy})\end{array}$ & $\begin{array}{c}F_{x}^{\mathrm{a}} \\
\left(\times 10^{-13} \mathrm{erg} \mathrm{s}^{-1} \mathrm{~cm}^{-2}\right)\end{array}$ & References \\
\hline J0030+0451 & 3.05 & $<1.0$ & 0.23 & 0.6 & 2.5 & 1,2 \\
$\mathrm{~J} 0218+4232$ & 2.32 & 7.78 & $>5.9$ & 0.9 & 1.5 & 3,4 \\
$\mathrm{~J} 0437-4715$ & 5.76 & 1.86 & $0.14^{\mathrm{b}}$ & 137 & 19. & $4,5,6$ \\
$\mathrm{~J} 0751+1807$ & 3.48 & 0.80 & 2.02 & 1.0 & 0.83 & 4,7 \\
$\mathrm{~J} 1012+5307$ & 5.26 & 0.97 & 0.84 & 2.8 & 0.49 & $4,7,8$ \\
$\mathrm{~J} 1024-0719$ & 5.16 & $<0.30$ & $<0.20^{\mathrm{b}}$ & 0.9 & 0.2 & $4,6,9$ \\
$\mathrm{~J} 1744-1134$ & 4.07 & 0.71 & $0.36^{\mathrm{b}}$ & 2.0 & 0.2 & $4,6,10$ \\
B1937+21 & 1.56 & 10.6 & $>3.6^{\mathrm{b}}$ & 16 & 3.7 & $7,9,11$ \\
B1957+20 & 1.61 & 1.15 & 1.53 & 0.35 & 3.0 & $4,7,9$ \\
$\mathrm{~J} 2124-3358$ & 4.93 & 1.30 & 0.25 & 2.6 & 2.5 & $4,6,9$ \\
B1821-24 (M28) & 3.05 & 162. & $5.5^{\mathrm{b}}$ & 1.9 & 5.2 & 4,7 \\
\hline
\end{tabular}

References. - 1 (Lommen et al. 2000); 2 (Becker et al. 2000); 3 (Navarro et al. 1995); 4 (BT99); 5 (van Straten et al. 2001); 6 (Toscano et al. 1998); 7 (Taylor et al. 1995); 8 (Lange et al. 2001); 9 (Toscano et al. 1999b); 10 (Toscano et al. 1999a); 11 (Takahashi et al. 2001).

${ }^{a}$ X-ray flux in ROSAT $(0.1-2.4 \mathrm{keV})$ band. Value for B1937+21 in ASCA (2-10 keV) band.

${ }^{\mathrm{b}}$ Accurate distances/limits. Other estimates are obtained primarily from dispersion measures together with the Taylor \& Cordes (1993) electron density model, and are rather uncertain.

TABLE 3

RESUlTS FROM KS TWO-SAMPLE COMPARISONS

\begin{tabular}{lccc}
\hline \hline & MSP & $\begin{array}{c}\text { Medium } \\
\text { (raw) }\end{array}$ & $\begin{array}{c}\text { Medium } \\
\text { (corr) }\end{array}$ \\
\hline Soft & 0.66 & 0.0034 & 0.11 \\
Medium (raw) & 0.16 & $\ldots$ & $\ldots$ \\
Medium (corr) & 0.82 & $\ldots$ & $\ldots$ \\
\hline
\end{tabular}

Note. - The entries give the probability of the null hypothesis that the two samples are drawn from the same underlying distribution. Values of less than 0.05 indicate significant differences between the samples. 
TABLE 4

Results FROM Generalized King Model Fits

\begin{tabular}{llrc}
\hline \hline \multicolumn{1}{c}{ Sample $^{\mathrm{a}}$} & $N$ & \multicolumn{1}{c}{$r_{c}\left({ }^{\prime \prime}\right)^{\mathrm{b}}$} & \multicolumn{1}{c}{$\alpha$} \\
\hline Soft & 44 & $15.2 \pm 3.5$ & $-3.6 \pm 0.8$ \\
Medium (raw) & 41 & $5.6 \pm 3.9$ & $-1.9 \pm 0.3$ \\
Medium (corr) & 33 & $9.0 \pm 6.9$ & $-2.5 \pm 1.0$ \\
MSP & 16 & $13.1 \pm 6.9$ & $-2.4 \pm 1.1$ \\
CV & 19 & $10.3 \pm 10.0$ & $-3.1 \pm 3.7$ \\
BY + RS c & 14 & $15.8 \pm 4.5$ & $-5.6 \pm 4.1$ \\
No ID & 22 & $17.5 \pm 3.6$ & $-3.1 \pm 0.7$ \\
\hline
\end{tabular}

${ }^{\text {a }}$ Samples were delimited by a radial cutoff of $250^{\prime \prime}$ except for the MSP group for which $100^{\prime \prime}$ was used.

${ }^{\mathrm{b}}$ Core radius for x-ray source population (vs. $r_{c}$ $=24.0^{\prime \prime}$ for the optical; see text).

${ }^{\mathrm{c}} \mathrm{BY}+\mathrm{RS}$ sample is the combination of BY Dra (main sequence) binaries and some possible RS CVn (sub-giant) systems.

TABLE 5

CANDIDATE MSPS FROM SOFT SOURCES

\begin{tabular}{cccc}
\hline \hline $\begin{array}{c}\mathrm{RA}^{\mathrm{a}} \\
(\mathrm{J} 2000)\end{array}$ & $\begin{array}{c}\mathrm{Dec}^{\mathrm{a}} \\
(\mathrm{J} 2000)\end{array}$ & $\begin{array}{c}\text { Medcts } \\
(0.5-4.5 \mathrm{keV})\end{array}$ & $\begin{array}{c}\log \left(\mathrm{L}_{x}\right)^{\mathrm{b}} \\
(0.5-2.5 \mathrm{keV})\end{array}$ \\
\hline 00:24:13.45(1) & $-72: 04: 51.27(8)$ & 33.5 & 30.7 \\
00:24:12.53(1) & $-72: 04: 41.13(5)$ & 8.3 & 30.1 \\
00:24:11.64(3) & $-72: 05: 03.72(8)$ & 9.1 & 30.1 \\
00:24:07.38(2) & $-72: 04: 49.41(5)$ & 41.8 & 30.8 \\
00:24:06.05(2) & $-72: 05: 01.7(1)$ & 12.7 & 30.3 \\
00:24:04.64(2) & $-72: 04: 34.5(1)$ & 18.7 & 30.5 \\
00:24:03.22(2) & $-72: 04: 29.74(8)$ & 15.7 & 30.4 \\
00:24:03.14(2) & $-72: 04: 26.55(9)$ & 16.7 & 30.4 \\
00:24:02.63(3) & $-72: 05: 38.5(1)$ & 13.8 & 30.3 \\
00:24:02.52(2) & $-72: 04: 44.1(1)$ & 20.4 & 30.5 \\
00:23:59.91(2) & $-72: 05: 04.7(1)$ & 21.2 & 30.5 \\
00:23:59.45(2) & $-72: 04: 47.9(2)$ & 9.4 & 30.2 \\
00:24:01.45(3) & $-72: 04: 41.6(1)$ & 9.7 & 30.2 \\
00:24:01.04(2) & $-72: 04: 13.9(1)$ & 12.5 & 30.3 \\
00:23:59.45(3) & $-72: 04: 38.5(1)$ & 9.9 & 30.2 \\
00:24:05.03(4) & $-72: 05: 02.8(1)$ & 9.0 & 30.1 \\
00:24:17.20(2) & $-72: 03: 03.2(1)$ & 8.7 & 30.1 \\
00:24:11.50(1) & $-72: 02: 24.7(1)$ & 20.6 & 30.5 \\
00:24:42.49(4) & $-72: 06: 20.6(2)$ & 15.1 & 30.4 \\
\hline
\end{tabular}

Note. - The first 5 source entries, and the 4th from the bottom of the table, are all particularly promising MSP candidates as they are all included in the Albrow et al. (2001) HST field and yet lack evidence for optical counterparts with either CV or BY Dra characteristics.

${ }^{a}$ The RA and Dec have been corrected to the MSP coordinate frame.

${ }^{\mathrm{b}} \mathrm{L}_{x}$ values calculated as for Table 1 . 